TRANSACTIONS OF THE

AMERICAN MATHEMATICAL SOCIETY

Volume 349, Number 6, June 1997, Pages 2317-2340

S $0002-9947(97) 01769-8$

\title{
ALGEBRAS ASSOCIATED TO ELLIPTIC CURVES
}

\author{
DARIN R. STEPHENSON
}

\begin{abstract}
This paper completes the classification of Artin-Schelter regular algebras of global dimension three. For algebras generated by elements of degree one this has been achieved by Artin, Schelter, Tate and Van den Bergh. We are therefore concerned with algebras which are not generated in degree one. We show that there exist some exceptional algebras, each of which has geometric data consisting of an elliptic curve together with an automorphism, just as in the case where the algebras are assumed to be generated in degree one.

In particular, we study the elliptic algebras $A(+), A(-)$, and $A(\mathbf{a})$, where $\mathbf{a} \in \mathbb{P}^{2}$, which were first defined in an earlier paper. We omit a set $S \subset \mathbb{P}^{2}$ consisting of 11 specified points where the algebras $A(\mathbf{a})$ become too degenerate to be regular.

Theorem. Let $A$ represent $A(+), A(-)$ or $A(\mathbf{a})$, where $\mathbf{a} \in \mathbb{P}^{2} \backslash S$. Then $A$ is an Artin-Schelter regular algebra of global dimension three. Moreover, $A$ is a Noetherian domain with the same Hilbert series as the (appropriately graded) commutative polynomial ring in three variables.
\end{abstract}

This, combined with our earlier results, completes the classification.

\section{INTRODUCTION}

M. Artin, J. Tate and M. Van den Bergh have introduced an important class of noncommutative graded $k$-algebras which can be defined via an elliptic curve $E$, an automorphism $\sigma$ of $E$ and an invertible sheaf $\mathcal{L}$ on $E$ of degree two or three [ATV1]. In this article, we study algebras which have a similar associated triple, $(E, \sigma, \mathcal{L})$, where in this case $\mathcal{L}$ has degree one.

Let $k$ be an algebraically closed field of characteristic zero. A $k$-algebra $A$ is said to be $\mathbb{N}$-graded if $A$ is a direct sum of $k$-vector spaces, $A=\bigoplus_{i \in \mathbb{N}} A_{i}$, such that $A_{i} A_{j} \subseteq A_{i+j}$ for all $i, j \in \mathbb{N}$. An $\mathbb{N}$-graded $k$-algebra $A$ is said to be connected graded if $A_{0}=k$ and each $A_{i}$ is a finite dimensional $k$-vector space. Artin-Schelter regular algebras, or AS-regular algebras, are defined to be connected graded $k$ algebras with finite global dimension with polynomial growth which satisfy a version of the Gorenstein property which will be given formally in section 5 .

In [St2], we show that AS-regular algebras of global dimension three which are not generated by elements of degree one fall into one of several classes. Then we

Received by the editors November 14, 1995.

1991 Mathematics Subject Classification. Primary 16W50, 14A22, 16P40; Secondary 16P90, $16 \mathrm{E} 10$.

Key words and phrases. Graded ring, Artin-Schelter regular algebra, noncommutative projective geometry, elliptic algebra, point module.

This research was supported in part by a graduate research fellowship on NSF grant number 9304423

(C)1997 American Mathematical Society 
proceed to show exactly which members of each class are AS-regular except in two cases. In these remaining cases the algebras become more complicated, and it is impossible to study them using traditional ring-theoretic techniques. In this article, we employ generalizations of the geometric techniques of [ATV1] to these algebras and determine precisely which algebras are AS-regular, thus completing the classification.

We now proceed with the definition of these algebras in terms of generators and relations. As will become evident after we have stated the main results, a more natural definition arises from the fact that these algebras are completely determined by the associated geometric data. Let $T=k\{x, y\}$ be the free algebra, graded so that $\operatorname{deg}(x)=1$ and $\operatorname{deg}(y)=2$. The algebras we will study in this article may be presented as quotients of $T$ by an ideal $I=\left(f_{1}, f_{2}\right)$, where $\operatorname{deg}\left(f_{1}, f_{2}\right)=(5,4)$. Let $\mathbf{a}=(a: b: c) \in \mathbb{P}_{k}^{2}$. We define algebras

$$
A(\mathbf{a})=T /\left(f_{1}, f_{2}\right)
$$

where

$$
\begin{aligned}
& f_{1}=a y^{2} x+c y x y+a x y^{2}+b x^{5}, \\
& f_{2}=a x^{2} y+c x y x+a y x^{2}+b y^{2} .
\end{aligned}
$$

Also, we define two more algebras:

$$
A( \pm)=T /\left(f_{1}, f_{2}\right)
$$

where

$$
\begin{aligned}
& f_{1}=x y^{2}+2 x y x^{2} \pm i y^{2} x \pm 2 i y x^{3}, \\
& f_{2}=x^{2} y+y x^{2}+y^{2} .
\end{aligned}
$$

As one might expect, for some values of the parameter, the algebras $A(\mathbf{a})$ become too degenerate to be AS-regular. This occurs for a in the following subset of $\mathbb{P}_{k}^{2}$, consisting of 11 points.

$$
\begin{aligned}
S= & \{(0: 0: 1),(0: 1: 0)\} \cup\left\{(1: b:-1) \mid b^{3}=-1\right\} \\
& \cup\left\{(1: b: c) \mid c=(1 \pm \sqrt{5}) / 2, b^{3}=-c^{-2}\right\},
\end{aligned}
$$

Our main result is the following, which is proven in section 5 .

Theorem 1.1. Let $A$ represent either $A( \pm)$ or $A(\mathbf{a})$, where $\mathbf{a} \notin S$. Then

(i) A is AS-regular of global dimension three.

(ii) $A$ is a Noetherian domain.

(iii) $A$ has the same Hilbert series as the polynomial ring $k\left[x_{1}, x_{2}, x_{3}\right]$ graded so that $\operatorname{deg}\left(x_{i}\right)=i$.

Moreover, if $\mathbf{a} \notin S$, then $A(\mathbf{a})$ is not $A S$-regular.

The proof of Theorem 1.1 involves the description of the algebras in terms of the associated geometry, which is also of interest. As in [ATV1], one of the most useful notions is that of a point module. Let $M$ be a graded right module over $A( \pm)$ or $A(\mathbf{a})$. Then $M$ is a point module if $M$ is generated by $M_{0}+M_{1}, \operatorname{dim} M_{i}=1$ for all $i \geq 0$, and every proper graded factor module of $M$ is finite dimensional. This definition is slightly different than that of Artin, Tate and Van den Bergh, but becomes the same when the algebras are generated in degree one.

In section 2 , we prove the following result which establishes the connection to algebraic geometry. 
Theorem 1.2. Let $\Gamma$ be the set of isomorphism classes of point modules over $A(\mathbf{a})$, where $\mathbf{a} \in \mathbb{P}^{2} \backslash S$ and $b \neq 0$. Then $\Gamma$ has the structure of a (possibly reducible) projective variety. This variety is isomorphic to the graph of an automorphism $\sigma$ of a projective variety $V=V(\mathbf{a})$.

Remark. The following table gives the possibilities for the variety $V$ and the automorphism $\sigma$.

\begin{tabular}{|c|c|}
\hline a & $V=V(\mathbf{a})$ \\
\hline$a=0$ and $b \neq-c^{3}$ & $V$ is a curve with 6 components \\
\hline$a=-c$ & $\sigma$ acts as a 6 -cycle on the components \\
\hline$b^{3} c=(c-a)^{2}\left(a^{2}-c^{2}\right)$ & $\begin{array}{c}V \text { is a curve with } 3 \text { components } \\
\sigma \text { acts as an } 3 \text {-cycle on the components }\end{array}$ \\
\hline$b^{3}=(c-2 a)^{2}\left(c^{2}-a^{2}\right)$ & $\begin{array}{l}V \text { is a curve with } 2 \text { components } \\
\sigma \text { interchanges the components }\end{array}$ \\
\hline$b^{3}=(-c+2 a)^{3}$ & $V$ is an irreducible, singular curve \\
\hline all other $\mathbf{a}$ & $\begin{array}{l}V \text { is a smooth elliptic curve and } \\
\sigma \text { is translation by a point on } V \\
\text { via the group structure }\end{array}$ \\
\hline
\end{tabular}

A similar result holds for the algebras $A( \pm)$ :

Theorem 1.3. Let $\Gamma$ be the set of isomorphism classes of point modules over $A( \pm)$. Then $\Gamma$ has the structure of a projective variety which is isomorphic to the graph of an automorphism $\sigma$ of a smooth irreducible elliptic curve $E$. In this case, $\sigma$ is complex multiplication on $E$ of order 4 .

The key to proving the regularity of the algebras in question is finding factor rings whose study is possible using algebraic geometry. If $V$ is a projective variety defined over $k, \sigma$ is an automorphism of $V$, and $\mathcal{L}$ is an invertible sheaf on $V$ of positive degree, then we can form a connected graded $k$-algebra $B(V, \sigma, \mathcal{L})$ as in [ATV1]. We give the full definition in section 4.

Theorem 1.1 follows from the following result.

Theorem 1.4. Let $A=A( \pm)$ or $A(\mathbf{a})$ where $\mathbf{a} \notin S$ and $b \neq 0$. Let $(V, \sigma)$ be the geometric data given by the point modules over $A$. There exist a degree one invertible sheaf $\mathcal{L}$ on $V$ and a nonzero degree six normal element $d \in A$ such that

$$
\frac{A}{d A} \cong B(V, \sigma, \mathcal{L})
$$

To give the intuition for the above results, it is first necessary to review the construction of the algebra $A$ when $V=E$ is an elliptic curve and $\operatorname{deg}(\mathcal{L})=2$ as in [ATV1]. First suppose that $\operatorname{deg}(\mathcal{L})=2$ and $\sigma=1$. Then

$$
B=B(E, \sigma, \mathcal{L}) \cong k[x, y, z] /(f) \cong k\{x, y, z\} /\left(r_{1}, r_{2}, r_{3}, f\right),
$$

where $\operatorname{deg}(x, y, z)=(1,1,2), f$ is a degree four polynomial and $r_{1}, r_{2}$ and $r_{3}$ are the commutation relations. If, on the other hand, $\sigma$ is generic, then

$$
B \cong k\{x, y\} /\left(f_{1}, f_{2}, f_{3}\right),
$$

where $\operatorname{deg}(x, y)=(1,1)$ and $\operatorname{deg}\left(f_{1}, f_{2}, f_{3}\right)=(3,3,4)$. Hence, $B$ can be presented with one fewer generator and one fewer relation than in the commutative case. The generator and relation of degree two do not occur. Then the algebra $A(E, \sigma, \mathcal{L})$ is defined to be $k\{x, y\} /\left(f_{1}, f_{2}\right)$. 
Now assume $\operatorname{deg}(\mathcal{L})=1$ and $\sigma=1$. Then

$$
B=B(E, \sigma, \mathcal{L}) \cong k[x, y, z] /(f) \cong k\{x, y, z\} /\left(r_{1}, r_{2}, r_{3}, f\right),
$$

where $\operatorname{deg}(x, y, z)=(1,2,3)$ and $f$ is a degree six polynomial. Now let $\sigma$ be generic. One might expect that again $B$ can be presented with one fewer generator and one fewer relation than in the commutative case, that is,

$$
B \cong k\{x, y\} /\left(f_{1}, f_{2}, f_{3}\right),
$$

where $\operatorname{deg}(x, y)=(1,2)$ and $\operatorname{deg}\left(f_{1}, f_{2}, f_{3}\right)=(4,5,6)$. Also, one might expect that the algebra $A=k\{x, y\} /\left(f_{1}, f_{2}\right)$ is an Artin-Schelter regular algebra of global dimension three. Our results show that this intuition is correct.

\section{Point Modules}

We work in the category of $\mathbb{Z}$-graded right $A$-modules. If $M=\bigoplus_{i \in \mathbb{Z}} M_{i}$ is a graded $A$-module and $z$ is an integer, we use $M[z]$ to denote the shifted module for which $M[z]_{i}=M_{i+z}$, and we use $M_{\geq z}$ to denote the 'tail' $\bigoplus_{i \geq z} M_{i}$.

In [ATV1], a point module over a graded algebra generated in degree one is defined to be a graded right module $M=\bigoplus M_{i}$ such that (i) $M$ is generated by $M_{0}$, (ii) $M$ is 1 -critical, and (iii) $\operatorname{dim}_{k} M_{i}=1$ for $i \geq 0$. In the case that $A$ is not generated in degree one, this definition is not general enough, since 'gaps' can occur in the Hilbert series of a cyclic module. Thus, we are led to relax condition (iii) to (iii)' $\operatorname{dim} M_{i} \leq 1$ for $i \geq 0$. The resulting definition is equivalent to that in [ATV1] when the algebra is generated in degree one. When the algebra is not generated in degree one, this new definition is somewhat hard to work with, since the truncated shift $M[1]_{\geq 0}$ of a point module need not be a point module. For the algebras we are interested in, this definition is equivalent (in the proper sense) to the following, which we take as our definition.

Definition 2.1. Let $M$ be a graded right module over $A(\mathbf{a})$ or $A( \pm)$. Then $M$ is a point module if

(i) $M=M_{0} A+M_{1} A$,

(ii) $\operatorname{dim} M_{i}=1$ for $i \geq 0$,

(iii) $M$ is 1 -critical.

We should point out that this definition is only well-suited to the particular algebras in question, not to all connected graded algebras which are not generated in degree one.

The aim of this section is to show that for $A=A( \pm)$ or $A(\mathbf{a})$ with $\mathbf{a} \in \mathbb{P}^{2} \backslash S$ and $b \neq 0$, the set of point modules over $A$ has the structure of the graph of an automorphism of a projective variety, where the automorphism comes from the shift functor on $A$-modules. In later sections, we use this geometric data to prove the regularity of $A$.

For the remainder of this section we will assume that $A$ is either $A( \pm)$ or $A(\mathbf{a})$. Define $\Gamma$ to be the set of isomorphism classes of point modules over $A$. When we wish to clarify which $\Gamma$ we are dealing with, we will use the notation $\Gamma( \pm)$ or $\Gamma(\mathbf{a})$. We will also need the following definition:

Definition 2.2. A graded right $A$ module $M$ is a truncated point module of length $d$ if

(i) $M=M_{0} A+M_{1} A$, 
(ii) $\operatorname{dim} M_{i}= \begin{cases}1 & \text { if } 0 \leq i \leq d-1, \\ 0 & \text { otherwise, }\end{cases}$

(iii) $\operatorname{soc}(M) \subseteq M_{d-2} \oplus M_{d-1}$.

Let $\Gamma_{d}$ be the set of isomorphism classes of truncated point modules of length $d+1$ over $A$. The following proposition justifies the name 'truncated point module.'

Lemma 2.3. For all $d>1$ we have a function $\pi_{d}: \Gamma_{d} \rightarrow \Gamma_{d-1}$ induced by $M \mapsto$ $M / M_{d}$. We also have a function $\rho_{d}: \Gamma \rightarrow \Gamma_{d-1}$ induced by $M \mapsto M / M_{\geq d}$. These functions satisfy $\pi_{d} \rho_{d+1}=\rho_{d}$, and as a set, $\Gamma=\lim \Gamma_{d}$.

Proof. For ease of notation, we will use $x$ and $y$ to represent the images of the generators of $T$ in $A$. Let $M$ be a truncated point module of length $d$ and let $N=M / M_{\geq d-1}$. To show that $N$ is a truncated point module of length $d-1$, it clearly suffices to show that $\operatorname{soc}(N) \subseteq N_{d-3} \oplus N_{d-2}$. Let $U$ be a graded simple submodule of $N$. The module $U$ is one dimensional, and so $U=N_{i}$ for some $i$. Also, $N_{i} x=N_{i} y=0$. Therefore, $M_{i} x \subseteq M_{\geq d-1}$ and $M_{i} y \subseteq M_{\geq d-1}$. If $i<d-3$ then $M_{i} x=M_{i} y=0$, contradicting the condition on the socle of $M$.

Similarly, let $M$ be a point module and let $N=M / M_{\geq d-1}$. Let $U=N_{i}$ be a graded simple submodule of $N$. If $i<d-3$, then $M_{i}$ is a graded submodule of $M$. But then $M / M_{i}$ is infinite dimensional, contradicting the fact that $M$ is 1-critical.

The fact that $\pi_{d} \rho_{d+1}=\rho_{d}$ is clear from the definitions. The fact that $\Gamma$ is the inverse limit of the $\Gamma_{d}$ follows from the fact that a point module is completely determined by its truncations.

We show that for $d \geq 5$, each of the sets $\Gamma_{d}$ naturally has the structure of a projective variety. We start by establishing a bijection between elements of $\Gamma_{d}$ and the orbits of an algebraic torus acting on a quasi-affine variety.

2.1. Point modules over $A(\mathbf{a})$ - general case. Suppose that $A=A(\mathbf{a})$ and let $d \geq 1$. Let $\left(x_{0}, \ldots, x_{d-1}, y_{0}, \ldots, y_{d-2}\right)$ be coordinates for $\mathbb{A}_{k}^{2 d-1}$. Let $U_{d}=U_{d}(\mathbf{a})$ be the locally closed subset of $\mathbb{A}_{k}^{2 d-1}$ defined by the following conditions:

$$
\begin{aligned}
a y_{i} y_{i+2} x_{i+4}+c y_{i} x_{i+2} y_{i+3}+a x_{i} y_{i+1} y_{i+3}+b x_{i} x_{i+1} x_{i+2} x_{i+3} x_{i+4} & =0, \\
0 & \leq i \leq d-5,
\end{aligned}
$$

$$
a x_{i} x_{i+1} y_{i+2}+c x_{i} y_{i+1} x_{i+3}+a y_{i} x_{i+2} x_{i+3}+b y_{i} y_{i+2}=0, \quad 0 \leq i \leq d-4,
$$

Also, define an action of a $d$-dimensional algebraic torus $\Lambda_{d}$ on $\mathbb{A}_{k}^{2 d-1}$ by

$$
\begin{aligned}
& \left(\lambda_{0}, \ldots, \lambda_{d-1}\right) \cdot\left(x_{0}, \ldots, y_{d-2}\right) \\
& \quad=\left(\lambda_{0} x_{0}, \ldots, \lambda_{i} x_{i}, \ldots \lambda_{d-1} x_{d-1}, \lambda_{0} \lambda_{1} y_{0}, \ldots, \lambda_{i} \lambda_{i+1} y_{i}, \ldots, \lambda_{d-2} \lambda_{d-1} y_{d-2}\right) .
\end{aligned}
$$

Lemma 2.1.4. For $d \geq 1$, there is a 1-1 correspondence between $\Gamma_{d}(\mathbf{a})$ and the orbits of $\Lambda_{d}$ on $U_{d}(\mathbf{a})$.

Proof. For a truncated point module $M$ of length $d+1$, let $m_{i}$ be a $k$-basis for $M_{i}$. Define $x_{i}, 0 \leq i \leq d-1$, and $y_{i}, 0 \leq i \leq d-2$, to be the scalars such that $m_{i} x=m_{i+1} x_{i}$ and $m_{i} y=m_{i+2} y_{i}$. It is clear that the conditions (2.1.2) and (2.1.1) hold for the point $\left(x_{0}, \ldots, y_{d-2}\right)$, since the relations defining $A$ must act as zero 
on $M$. As $M$ is generated in degrees zero and one, we see that $y_{i}=0$ implies that $x_{i+1} \neq 0$. As $\operatorname{soc}(M) \subseteq M_{d-1} \oplus M_{d}$, we see that $y_{i}=0$ implies $x_{i} \neq 0$. This shows that (2.1.3) also holds, and so $\left(x_{0}, \ldots, y_{d-2}\right)$ is a point of $U_{d}$. It is easily seen that the orbit of the point so defined does not depend on the basis of $M$ chosen above, and that the point depends only on the isomorphism class of $M$.

Conversely, any $(2 d-1)$-tuple of scalars $\left(x_{0}, \ldots, y_{d-2}\right)$ satisfying $(2.1 .2),(2.1 .1)$ and (2.1.3) can be used to define a truncated point module. For $0 \leq i \leq d-1$, let $M_{i}$ be a 1-dimensional $k$-vector space with basis $m_{i}$, and set $M=\bigoplus_{i=0}^{d} M_{i}$. Then define the action of $x$ and $y$ on $M$ by $m_{i} x=m_{i+1} x_{i}$ and $m_{i} y=m_{i+2} y_{i}$. We set $M_{d} x=M_{d} y=M_{d-1} y=0$. Since (2.1.2) and (2.1.1) hold, the relations defining $A$ act as zero on $M$, and so $M$ is indeed a graded $A$-module. The fact that (2.1.3) holds gives that $M$ is generated by $M_{0} \oplus M_{1}$ and that $\operatorname{soc}(M) \subseteq M_{d-1} \oplus M_{d}$. It is easy to see that points of $U_{d}$ in the same orbit define isomorphic truncated point modules.

We now proceed to show that there is a natural bijection between the set $\Gamma_{d}$ and the points of a projective variety $V_{d}$. For the following reasons, we omit the case $b=0$ from this discussion. The algebras $A(a: 0: c)$ can be regraded so that $\operatorname{deg}(x, y)=(1,1)$, and so they are classified by the results of [ATV1]. Also, the algebras $A(a: 0: c)$ are Ore extensions of AS-regular algebras of global dimension two (see Lemma 2.5.1), and therefore can be studied by less complicated techniques. Also, there are added technicalities in some cases, so for the rest of this subsection we will assume the following.

Hypothesis 2.1.5. Let $\mathbf{a}=(a: b: c) \in \mathbb{P}^{2}$ be chosen so that $\mathbf{a} \notin S$ and $a, b, c$ and $a+c$ are nonzero.

Let $\mathbf{P}_{d}=\mathbb{P}^{1} \times \cdots \times \mathbb{P}^{1}$ be the product of $d-1$ copies of $\mathbb{P}^{1}$, and let $\left(w_{i-1}, z_{i-1}\right)$ be homogeneous coordinates for the $i$ th copy of $\mathbb{P}^{1}$. Let $g_{d}: U_{d} \rightarrow \mathbf{P}_{d}$ be the $\Lambda_{d^{-}}$ invariant morphism such that the image of the point $\left(x_{0}, \ldots, x_{d-1}, y_{0}, \ldots, y_{d-2}\right)$ is the point of $\mathbf{P}_{d}$ defined by $w_{i}=x_{i} x_{i+1}$ and $z_{i}=y_{i}$.

For $0 \leq p \leq d-4$, define

$$
\begin{aligned}
& F_{1}^{p}=a w_{p} w_{p+1} z_{p+2}+c w_{p} z_{p+1} w_{p+2}+a z_{p} w_{p+1} w_{p+2}+b z_{p} w_{p+1} z_{p+2}, \\
& \begin{aligned}
F_{2}^{p}= & \left(a^{2} c-c^{3}\right) z_{p} w_{p+1} z_{p+2}+a b c w_{p} w_{p+1} w_{p+2}+\left(a b c+a^{2} b\right) z_{p} z_{p+1} z_{p+2} \\
& \quad+b^{2} c w_{p} z_{p+1} w_{p+2}+\left(a^{3}-a c^{2}\right) z_{p} z_{p+1} w_{p+2}+\left(a^{3}-a c^{2}\right) w_{p} z_{p+1} z_{p+2} .
\end{aligned}
\end{aligned}
$$

We define $V_{d}$ to be the closed subset of $\mathbf{P}_{d}$ defined by the simultaneous vanishing of the forms $F_{j}^{p}$ for $j=1,2$ and $0 \leq p \leq d-4$.

Proposition 2.1.6. Let $A=A(\mathbf{a})$ where a satisfies Hypothesis 2.1.5. The image of $U_{d}$ under $g_{d}$ is $V_{d}$. Moreover, for each $p \in V_{d}$ the fiber $g^{-1}(p)$ is a $\Lambda_{d}$-orbit on $U_{d}$.

Proof. The proof amounts to easy but tedious linear algebra using (2.1.1), (2.1.2) and (2.1.3). We give a brief outline here, to provide the interested reader with enough details to complete the proof.

The expression $F_{1}^{p}$ clearly comes directly from (2.1.2), and thus it is clear that for any $u \in U_{d}$, we have $F_{1}^{p}\left(g_{d}(u)\right)=0$. The expression $F_{2}^{p}$ comes from the fact that the equations (2.1.1) for $i=p$ and (2.1.2) for $i=p+1$ can be written in 
matrix form as $N\left(\begin{array}{l}y_{p+3} \\ x_{p+4}\end{array}\right)=0$, where

$$
N=\left(\begin{array}{cc}
c y_{p} x_{p+2}+a x_{p} y_{p+1} & a y_{p} y_{p+2}+b x_{p} x_{p+1} x_{p+2} x_{p+3} \\
a x_{p+1} x_{p+2}+b y_{p+1} & c x_{p+1} y_{p+2}+a y_{p+1} x_{p+3}
\end{array}\right) .
$$

Thus, if $\left(\ldots, x_{p}, \ldots, y_{p+3}, \ldots\right) \in U_{d}$, then $\operatorname{det}(N)=0$, and this last equation can be shown to imply $F_{2}^{p}=0$. From this it follows that $g_{d}(u) \in V_{d}$.

The rest of the proof is straightforward.

We use this bijection to give $\Gamma_{d}$ the structure of a projective variety, and from now on we will identify $\Gamma_{d}$ with $V_{d}$. For $0 \leq i \leq j \leq d-2$, let $p r_{i, j}: \mathbf{P}_{d} \rightarrow \mathbf{P}_{j-i+1}$ be the projection mapping onto the $i$ th through the $j$ th copies of $\mathbb{P}^{1}$. It is easy to see that $p r_{0, d-3}\left(V_{d}\right) \subseteq V_{d-1}$, and that this morphism corresponds to the truncation map $\pi_{d}: \Gamma_{d} \rightarrow \Gamma_{d-1}$. This allows us to define the structure of a variety on $\Gamma=\lim \Gamma_{d}$. We show now that for all $d \geq 5$, there is an isomorphism of varieties $V_{d} \rightarrow V_{4}$

Lemma 2.1.7. Let $A=A(\mathbf{a})$ where a satisfies Hypothesis 2.1.5. If $d \geq 5$, the map pr $_{0, d-3}$ restricted to $V_{d}$ is an isomorphism from $V_{d}$ to $V_{d-1}$.

Proof. The morphism $p_{d}=\left.p r_{0, d-3}\right|_{V_{d}}$ is a bijection if and only if the equations $F_{i}^{d-4}=0$ have a solution which is unique up to scalar multiple, for all choices of $\left[\left(w_{0}: z_{0}\right), \ldots,\left(w_{d-3}: z_{d-3}\right)\right] \in V_{d-1}$. This occurs if and only if the matrix

$$
M=\left(\begin{array}{ll}
m_{11} & m_{12} \\
m_{21} & m_{22}
\end{array}\right)
$$

where

$$
\begin{aligned}
& m_{11}=c w_{d-4} z_{d-3}+a z_{d-4} w_{d-3}, \\
& m_{12}=a w_{d-4} w_{d-3}+b z_{d-4} w_{d-3}, \\
& m_{21}=a b c w_{d-4} w_{d-3}+b^{2} c w_{d-4} z_{d-3}+\left(a^{3}-a c^{2}\right) z_{d-4} z_{d-3}, \\
& m_{22}=\left(a^{2} c-c^{3}\right) z_{d-4} w_{d-3}+\left(a b c+a^{2} b\right) z_{d-4} z_{d-3}+\left(a^{3}-a c^{2}\right) w_{d-4} z_{d-3},
\end{aligned}
$$

has rank one at every point of $V_{d-1}$.

The equations $F_{i}^{d-5}=0$ hold in $V_{d-1}$, since $d \geq 5$. It is easily checked that these equations can be rewritten as $\left(w_{d-5}, z_{d-5}\right) M=0$. The scalars $w_{d-5}$ and $z_{d-5}$ cannot both be zero, and so $\operatorname{det} M=0$. Thus $M$ has rank at most one.

Suppose $M$ has rank zero. Then each entry of $M$ is zero. The fact that $a w_{d-4} w_{d-3}+b z_{d-4} w_{d-3}=0$ gives that either $w_{d-3}=0$ or $z_{d-4}=-a b^{-1} w_{d-4}$. If $w_{d-3}=0$, we must have $w_{d-4}=0$ and either $z_{d-3}$ or $z_{d-4}=0$, a contradiction.

So $w_{d-3} \neq 0$, and we may set $w_{d-3}=1$. Also, $z_{d-4}=-a b^{-1} w_{d-4}$, which implies that $w_{d-4}$ and $z_{d-4}$ are nonzero. We may assume that $w_{d-4}=1$ and $z_{d-4}=-a b^{-1}$. The fact that $c w_{d-4} z_{d-3}+a z_{d-4} w_{d-3}=0$ now gives that $z_{d-3}=-a^{2} b^{-1} c^{-1}$. Expanding the entries in the second row of $M$ gives that $c^{2}-a c+a^{2}=0$ and $\left(a^{4}+a^{3} c-b^{3}\right)=0$. These two equations imply that $(a: b: c)=(1: b: c)$, where $c=(1 \pm \sqrt{5}) / 2$ and $b^{3}=-c^{-2}$. This shows that $(a: b: c) \in S$, a contradiction.

This shows that $p_{d}$ is a bijection. The fact that the $p_{d}^{-1}$ is a morphism of varieties comes from the fact that it is defined locally by the rows of the matrix $M$.

In the same way, the function $q_{d}=\left.p r_{1, d-2}\right|_{V_{d}}$ is an isomorphism of $V_{d}$ onto $V_{d-1}$. Now the function $\sigma=p_{5}^{-1} q_{5}$ is an automorphism of the variety $V_{4}$. If $M$ is a point module with associated point $p(M) \in V_{4}$, then $M[1]_{\geq 0}$ is a point module and its associated point in $V_{4}$ is $\sigma(p(M))$. An explicit formula for $\sigma$ can easily be written down (see [St1]), but will not be needed here. 
We have the following nice description of the variety $V_{4}$.

Lemma 2.1.8. Suppose a satisfies Hypothesis 2.1.5. Then $p_{0,1}$ restricted to $V_{d}$ is an isomorphism onto its image, which is a curve on the quadric surface $\mathbb{P}^{1} \times \mathbb{P}^{1}$.

Proof. The proof of this result is identical to that of Lemma 2.1.7, and so we will omit it. The curve $E=E(\mathbf{a})$ on the quadric surface defined by the image of $V_{4}$ is given by the vanishing of the following form:

$$
\begin{aligned}
& f\left(w_{0}, w_{1}, z_{0}, z_{1}\right)=\operatorname{det} M \\
&=\left(c^{3}-a^{2} c+b^{3}\right) w_{0} w_{1} z_{0} z_{1}-\left(a b c+a^{2} b\right) w_{0} z_{0} z_{1}^{2} \\
&-\left(a b c+a^{2} b\right) w_{1} z_{0}^{2} z_{1}+a b^{2} w_{0} w_{1}^{2} z_{0}+a b^{2} w_{0}^{2} w_{1} z_{1} \\
&+\left(a c^{2}-a^{3}\right) w_{1}^{2} z_{0}^{2}+\left(a c^{2}-a^{3}\right) w_{0}^{2} z_{1}^{2}+a^{2} b w_{0}^{2} w_{1}^{2} .
\end{aligned}
$$

The automorphism $\sigma$ on $V_{4}$ induces an automorphism of $E$, which we also will call $\sigma$. The adjunction formula [Ha, Example V.1.5.2] gives that $E$ has arithmetic genus one. In the cases where $E$ is smooth and irreducible, it is an elliptic curve. For convenience, we will idenitfy the variety of point modules $\Gamma$ together with its automorphism with $(E(\mathbf{a}), \sigma)$.

The following proposition gives the conditions on a under which the curve $E$ is singular or reducible, and its proof is elementary.

Proposition 2.1.10. Suppose $\mathbf{a}=(a: b: c)$ satisfies Hypothesis 2.1.5. Then the curve $E=E(\mathbf{a})$ is reducible if and only if either $b^{3} c=-(c-a)^{2}\left(c^{2}-a^{2}\right)$ or $b^{3}=-(c-2 a)^{2}(c+a)$. Furthermore, if $E$ is irreducible, then it is singular if and only if $b^{3}=-(c+2 a)^{3}$. In this case, Sing $(E)$ consists of one point, which can be either a node or a cusp.

Remark. This is similar to what occurs in [ATV1], with one notable difference. In that paper, the curve is nonsingular whenever it in irreducible, whereas we can have an irreducible singular curve.

2.2. Point modules over $A(a: b: 0)$. If $\mathbf{a}=(a: b: 0)$, we take

$$
\begin{aligned}
& F_{1}^{p}=a z_{p} w_{p+1} z_{p+2} w_{p+3}+a w_{p} z_{p+1} w_{p+2} z_{p+3}+b w_{p} w_{p+1} w_{p+2} w_{p+3}, \\
& F_{2}^{p}=a w_{p} z_{p+2}+a z_{p} w_{p+2}+b z_{p} z_{p+2} .
\end{aligned}
$$

Then $V_{d} \subset \mathbf{P}_{d}$ is defined by the vanishing of $F_{1}^{p}$ for $0 \leq p \leq d-4$, and $F_{2}^{p}$ for $0 \leq p \leq d-5$.

Proposition 2.1.6 holds in this case, and Lemma 2.1.7 holds if and only if $d \geq 6$. Again, $p r_{0,1}$ and $p r_{1,2}$ give isomorphisms from $V_{d}$ to a curve $E$ of bidegree $(2,2)$ on $\mathbb{P}^{1} \times \mathbb{P}^{1}$, and $\sigma: p r_{0,1}^{-1} p r_{1,2}: E \rightarrow E$ is an automorphism of $E$. In fact, $E$ is defined by the equation (2.1.9) with $c=0$. The automorphism is a translation of order four.

2.3. Point modules over $A(0: b: c)$ and $A(a: b:-a)$. We assume $a=0$. The cases in which $c=-a$ are similar, and in fact, $A(0: b: 1) \cong A\left(-1: b^{4} /\left(1+b^{3}\right): 1\right)$ if $b^{3} \neq-1$. We also assume that $b^{3} \neq-c^{3}$, since the algebras with $a=0$ and $b^{3}=-c^{3}$ are Ore extensions (see Lemma 2.5.1), and thus can be studied in less complicated ways. 
Set

$$
\begin{aligned}
& F_{1}^{p}=w_{p+1}\left(c z_{p} z_{p+3}+b w_{p} w_{p+3}\right), \\
& F_{2}^{p}=w_{p+2}\left(c z_{p} z_{p+3}+b w_{p} w_{p+3}\right), \\
& F_{3}^{p}=z_{p+1}\left(c^{2} w_{p} w_{p+3}-b^{2} z_{p} z_{p+3}\right), \\
& F_{4}^{p}=z_{p+2}\left(c^{2} w_{p} w_{p+3}-b^{2} z_{p} z_{p+3}\right), \\
& F_{5}^{p}=w_{p} z_{p+1} w_{p+2} \\
& F_{6}^{p}=z_{p} w_{p+1} z_{p+2} .
\end{aligned}
$$

We define $V_{d} \subset \mathbf{P}_{d}$ by the simultaneous vanishing of $\left\{F_{j}^{p}: j=1,2,3,4\right.$ and $0 \leq$ $p \leq d-5\}$ and $\left\{F_{j}^{p}: j=5,6\right.$ and $\left.0 \leq p \leq d-4\right\}$.

Proposition 2.1.6 and Lemma 2.1.7 hold in this case. However, the map $p r_{0,1}$ does not give an isomorphism of $V_{4}$ with a curve on $\mathbb{P}^{1} \times \mathbb{P}^{1}$ as it does in other cases. The variety $V_{4}$ (which we will denote $E(0: b: c)$ ) is a reducible one-dimensional variety with six components. The automorphism $\sigma$ acts as a 6 -cycle on the components.

2.4. Point modules for $A( \pm)$. Let $A=A( \pm)$. We preserve the toral action on $\mathbb{A}_{k}^{2 d-1}$ from above. We take $U_{d}( \pm)$ to be the subset of $\mathbb{A}_{k}^{2 d-1}$ defined by the conditions:

$$
\begin{gathered}
x_{j} y_{j+1} y_{j+3}+2 x_{j} y_{j+1} x_{j+3} x_{j+4} \pm i y_{j} y_{j+2} x_{j+4} \pm 2 i y_{j} x_{j+2} x_{j+3} x_{j+4}=0, \\
\quad 0 \leq j \leq d-5, \\
\text { 4.2) } \quad x_{j} x_{j+1} y_{j+2}+y_{j} x_{j+2} x_{j+3}+y_{j} y_{j+2}=0, \quad 0 \leq j \leq d-4, \\
\quad y_{j}=0 \Longrightarrow x_{j} x_{j+1} \neq 0, \quad 0 \leq j \leq d-2,
\end{gathered}
$$

The following result is the analogue of Lemma 2.1.4 in this case, and its proof is identical.

Lemma 2.4.4. For $d \geq 1$, there is a 1-1 correspondence between $\Gamma_{d}( \pm)$ and the set of orbits of $\Lambda_{d}$ on $U_{d}( \pm)$.

Set

$$
\begin{aligned}
& F_{1}^{p}=w_{p} z_{p+1} w_{p+2} z_{p+3}+2 w_{p} z_{p+1} w_{p+2} w_{p+3} \pm i z_{p} w_{p+1} z_{p+2} z_{p+3} \\
& \quad \quad \pm 2 i z_{p} w_{p+1} w_{p+2} w_{p+3} \\
& F_{2}^{p}=w_{p} z_{p+2}+z_{p} w_{p+2}+z_{p} z_{p+2} .
\end{aligned}
$$

We define $V_{d}$ to be the subset of $\mathbf{P}_{d}$ defined by the simultaneous vanishing of the forms $F_{1}^{p}$ for $0 \leq p \leq d-5$ and $F_{2}^{p}$ for $0 \leq p \leq d-4$.

Proposition 2.1.6 and Lemma 2.1.7 hold in this case. Again, the projections $p r_{0,1}$ and $p r_{1,2}$ give isomorphisms from $V_{d}$ to a curve $E \subset \mathbb{P}^{1} \times \mathbb{P}^{1}$. The curve $E=E( \pm)$ is defined by

$$
\begin{aligned}
& f\left(w_{0}, w_{1}, z_{0}, z_{1}\right)=(2 \pm 2 i) w_{0} w_{1} z_{0} z_{1}+2 w_{0}^{2} w_{1} z_{1}+w_{0} z_{0} z_{1}^{2}+w_{0}^{2} z_{1}^{2} \\
& \quad \pm i w_{1}^{2} z_{0}^{2} \pm 2 i w_{0} w_{1}^{2} z_{0} \pm i w_{1} z_{0}^{2} z_{1} .
\end{aligned}
$$

Remark. It is easily checked that $E( \pm)$ are smooth irreducible curves. The adjunction formula [Ha, Example V.1.5.2] gives that both of the curves $E( \pm)$ have genus one. It is easily checked that $\sigma$ is an automorphism of order four of $E( \pm)$ with fixed points, and so is complex multiplication by a fourth root of unity. 
2.5. Preparatory results. The table in the introduction gives all of the possibilities for the curves $E(\mathbf{a})$ and $E( \pm)$. In all of the cases where $E$ is one-dimensional, it has arithmetic genus 1 by the adjunction formula. Also, if $\omega_{E}$ represents the canonical sheaf on $E$, then we have $\omega_{E} \cong \mathcal{O}_{E}$.

In a few cases, the algebras $A(\mathbf{a})$ are Ore extensions, and are easily studied by ring-theoretic methods. We give the following results for two of these cases, so that in the later sections we may concentrate on cases where the associated variety is a curve.

Lemma 2.5.1. Let $A=A(a: b: c)$ be such that $(a: b: c) \notin S$ and either (i) $a=0$ and $b^{3}=-c^{3}$, or (ii) $b=0$. Then there exists a graded subring $R$ of $A$ which is an AS-regular algebra of global dimension two, and $A$ is isomorphic to an Ore extension of the form $R[t ; \theta, \delta]$, where $\theta$ is an automorphism of $R$ and $\delta$ is a $\theta$-derivation.

Proof. Part (i) follows immediately from the results of [St2] after a change of generator $y \mapsto y-x^{2}$. Part (ii) is also proven in [St2].

Corollary 2.5.2. Under the above hypotheses, $A$ is an AS-regular algebra of global dimension three.

Proof. This follows from [LS, Lemma].

Remark. The algebras $A(\mathbf{a})$ such that $E(\mathbf{a})$ is singular and irreducible are also Ore extensions as in the proposition. However, we will not need this in what follows.

\section{Some Facts about Sheaves}

In order to prove the regularity of the algebras in question, we require several facts about invertible sheaves on the varieties of point modules. We will use $E$ to denote either $E( \pm)$ or $E(\mathbf{a})$, where $\mathbf{a}$ is chosen so that $E(\mathbf{a})$ is a curve.

In the case that $E$ has at most three components, we identify $E \subset \mathbb{P}^{1} \times \mathbb{P}^{1}$ with $\Gamma_{5}$ as in section 2. If $E$ has six components, we assume that $E \subset \mathbb{P}^{1} \times \mathbb{P}^{1} \times \mathbb{P}^{1}$. Then torus action on $U_{5}$ allows us to define the invertible sheaf $\mathcal{L}=\mathcal{O}(1,0,0,0,0)$ on $\Gamma_{5}$ (or on $E$ ) whose sections are homogeneous polynomials of degree $(1,0,0,0,0)$. Clearly, $H^{0}(\mathcal{L})=k x_{0}$. Also, $y_{0}$ is naturally an element of $H^{0}\left(\mathcal{L} \otimes \mathcal{L}^{\sigma}\right)$, and, by the definition of $\sigma, x_{0} \circ \sigma^{i}=x_{i}$ and $y_{0} \circ \sigma^{i}=y_{i}$.

Suppose $E$ has at most three components. Then, considered as an invertible sheaf on $E, \mathcal{L}$ is isomorphic to the sheaf $\mathcal{O}_{E}(P)$, where $P$ is the degree one Cartier divisor determined by the point $[(0: 1),(b: c-a)] \in E$. If $E$ has six components, the invertible sheaf $\mathcal{L}=\mathcal{O}(1,0,0,0,0)$ has multidegree $(1,-1,1,0,0,0)$.

Definition 3.1. For the remainder of this article, we will refer to the geometric data $(E, \sigma, \mathcal{L})$ constructed above as a triple.

The next section provides useful information about the twisted coordinate ring of a triple. Before proceeding with this, we must show that certain sheaves related to $\mathcal{L}$ are well-behaved.

First, we fix some notation. If $f: X \rightarrow Y$ is a morphism of sheaves and $\mathcal{L}$ is a sheaf of $\mathcal{O}_{Y}$-modules, then we will use the notation $f^{*} \mathcal{L}$ for the inverse image of $\mathcal{L}$ under $f$ as defined in [Ha, p. 110]. If $\sigma: X \rightarrow X$ is an automorphism of $X$, we use $\mathcal{L}^{\sigma}$ to denote $\sigma^{*} \mathcal{L}$. 
We will use the following standard notation:

$$
\begin{gathered}
H^{i}(\mathcal{M})=H^{i}(E, \mathcal{M}), \\
h^{i}(\mathcal{M})=\operatorname{dim}\left(H^{i}(\mathcal{M})\right), \\
\mathcal{M}^{-1}=\operatorname{Hom}_{\mathcal{O}_{E}}\left(\mathcal{M}, \mathcal{O}_{E}\right) .
\end{gathered}
$$

On an irreducible curve of genus 1 , the dimension of the space of global sections of every invertible sheaf is easy to compute using the Riemann-Roch Theorem, and the reader who is interested chiefly in the case of smooth elliptic curves may safely skip directly to section 4 . In the reducible cases, sheaves are not so nicely behaved, which necessitates the following definition from [ATV1].

Definition 3.2. Let $E$ be a curve of genus one. An invertible sheaf of $\mathcal{O}_{E}$-modules $\mathcal{M}$ will be called tame if $\mathcal{M}=\mathcal{O}_{E}$, or either $H^{0}(\mathcal{M})=0$ or $H^{1}(\mathcal{M})=0$.

We now show that the sheaves to be used in the next section are tame.

Proposition 3.3. Let $(E, \sigma, \mathcal{L})$ be a triple. Then $\mathcal{L}_{n}$ is generated by its global sections for $n \geq 2$. Moreover, the following sheaves are tame:

(i) $\mathcal{L}_{n}$ for $n \geq 1$,

(ii) $\mathcal{L}_{2}^{-1} \otimes \mathcal{L}_{n}$ for $n \geq 2$.

(iii) $\mathcal{L}_{2}^{-1} \otimes \mathcal{L}_{m}^{\sigma^{2}} \otimes\left(\mathcal{L}_{2}^{\sigma^{m+2}}\right)^{-1}$ for $m \geq 5$ and $m \neq 7$.

If $E$ has at most three components, then (iii) holds for $m=7$.

Proof. In the case that $E$ has two or three components,

$$
\mathcal{L}_{n} \cong \mathcal{O}_{E}\left(P+\sigma^{-1} P+\cdots+\sigma^{-n+1} P\right)
$$

is generated by its global sections if $n \geq 2$ by [ATV1, Proposition 7.13].

If $E$ has six components $C_{1}, \ldots, C_{6}$ which are cyclically permuted by $\sigma$, it is easily checked that $\operatorname{deg}_{C_{i}}\left(\mathcal{L}_{n}\right) \geq 0$ for each $i$ and $n \geq 2$. Then $\mathcal{L}_{n}$ is generated by its sections by an easy generalization of [ATV1, Proposition 7.13].

By [ATV1, Corollary, p.71], in order to prove (i) and (ii), it suffices to prove the result for $\mathcal{L}$ and $\mathcal{L}_{2}^{-1} \otimes \mathcal{L}_{n}^{\sigma^{2}}$ for $n=2,3$. The verification of this fact is easy, using [ATV1, Proposition 7.12(i), (iii)] to examine the various cases.

Part (iii) also follows from checking the degrees explicitly and using [ATV1, Proposition 7.12(i),(iii)]. We note that [ATV1, Proposition 7.12(i)] fails to apply when $E$ has six components and $m=7$.

Proposition 3.4. Let $(E, \sigma, \mathcal{L})$ be a triple such that $E$ has two or three components. Then

(i) The sheaf $\mathcal{L}_{2}^{-1} \otimes \mathcal{L}_{n}^{\sigma^{2}}$ is generated by its global sections for $n=4,5$.

(ii) The multiplication map

$$
\mu_{1, n}: H^{0}(\mathcal{L}) \otimes H^{0}\left(\mathcal{L}_{n}\right) \rightarrow H^{0}\left(\mathcal{L}_{n+1}\right)
$$

is injective for $n=1,2,3$, as are the maps $\mu_{n, 1}$.

(iii) The multiplication map

$$
H^{0}\left(\mathcal{L}_{2}^{-1} \otimes \mathcal{L}_{n}^{\sigma^{2}}\right) \otimes H^{0}\left(\mathcal{L}^{\sigma^{n+2}}\right) \rightarrow H^{0}\left(\mathcal{L}_{2}^{-1} \otimes \mathcal{L}_{n+1}^{\sigma^{2}}\right)
$$

is injective if $n=3,4,5$.

Moreover, part (ii) holds in the six component case. 
Proof. Part (i) follows from [ATV1, Proposition 7.13].

The only way that the map in (ii) could have nonzero kernel is if some section of $\mathcal{L}$ vanishes on a component of $E$. As $H^{0}(\mathcal{L})=k x_{0}$, we can see by inspection that this is not the case when $E$ has two or three components, and so (ii) holds. The proof of (iii) is similar.

In the six component case, $x_{0}$ does vanish on one component of $E$. However, for $n=1,2,3$, global sections of $\mathcal{L}_{n}$ vanish on at most $n$ components of $E$. Thus, products of nonzero sections, cannot vanish on all six components of $E$.

We also require the following fact:

Lemma 3.5. For $i=1,2,3, \mathcal{L} \neq \mathcal{L}^{\sigma^{i}}$.

Proof. In the case that $E$ has at most three components, this is equivalent to $\sigma^{-i}(P) \neq P$. This is easy to see using the explicit form of $\sigma$ given in [St1].

In the six component case, the result is clear because $\mathcal{L}$ and $\mathcal{L}^{\sigma^{i}}$ have different multidegree.

\section{The Geometric Ring}

In this section, we define the twisted homogeneous coordinate ring and develop results necessary for proving the regularity of the associated algebra $A$.

Let $V$ be a variety defined over $k$, and let $\sigma$ be an automorphism of $V$. Let $\mathcal{L}$ be an invertible sheaf on $E$. For $n>0$, let $\mathcal{L}_{n}=\mathcal{L} \otimes \mathcal{L}^{\sigma} \otimes \cdots \otimes \mathcal{L}^{\sigma^{n-1}}$, where $\mathcal{L}^{\sigma}=\sigma^{*} \mathcal{L}$. Also, set $\mathcal{L}_{0}=\mathcal{O}_{V}$. Let $B_{n}=H^{0}\left(V, \mathcal{L}_{n}\right)$ and let $B=B(V, \sigma, \mathcal{L})=\bigoplus_{n=0}^{\infty} B_{n}$. For each $m, n \in \mathbb{N}$, we have an isomorphism $\mathcal{L}_{n} \otimes \mathcal{L}_{m}^{\sigma^{n}} \cong \mathcal{L}_{m+n}$, which gives rise to a map $\mu_{n, m}: B_{n} \otimes B_{m} \rightarrow B_{n+m}$ which is the composition of the obvious maps

$$
B_{n} \otimes B_{m}=H^{0}\left(\mathcal{L}_{n}\right) \otimes H^{0}\left(\mathcal{L}_{m}\right) \cong H^{0}\left(\mathcal{L}_{n}\right) \otimes H^{0}\left(\mathcal{L}_{m}^{\sigma^{n}}\right) \rightarrow H^{0}\left(\mathcal{L}_{m+n}\right)=B_{n+m} .
$$

If we consider elements $f \in B_{n}$ and $g \in B_{m}$ as rational functions on $V$, then $\mu_{n, m}(f, g)=f g^{\sigma^{n}}$, where $g^{\sigma^{n}}=g \circ \sigma^{n}$. This multiplication makes $B$ into a connected graded $k$-algebra called the twisted homogeneous coordinate ring of $(V, \sigma, \mathcal{L})$.

Theorem 4.1. Let $B=B(E, \sigma, \mathcal{L})$ be the twisted homogeneous coordinate ring of a triple (as defined by Definition 3.1). Then

(i) $B_{0}=k$, and $\operatorname{dim}_{k}\left(B_{n}\right)=n$ for $n \geq 1$.

(ii) $B$ is Noetherian, and the socle of $B$ as a left or right $B$-module is zero.

(iii) For $n \geq 3, B_{2} B_{n}=B_{n+2}$. If $\mathcal{L}_{2}^{-1} \otimes \mathcal{L}_{2}^{\sigma^{2}} \neq \mathcal{O}_{E}$, then also $B_{2} B_{2}=B_{4}$.

(iv) $B$ is generated by one element of degree one and one element of degree two.

(v) If $B$ is presented as a graded quotient algebra of the free algebra $T=k\{x, y\}$ graded so that $\operatorname{deg}(x)=1$ and $\operatorname{deg}(y)=2$ and $J \subset T$ is the ideal defining $B$, then $J=\left(j_{4}, j_{5}, j_{6}\right)$ where $\operatorname{deg}\left(j_{4}, j_{5}, j_{6}\right)=(4,5,6)$.

Remark. 1. To avoid excessive hypotheses in the reducible cases, we have stated Theorem 4.1 only for our specific definition of a triple. We remark, however, that the theorem holds more generally for any set of data $(E, \sigma, \mathcal{L})$ where $E$ is a smooth, irreducible elliptic curve, $\sigma$ is an automorphism of $E$, and $\mathcal{L}$ is a degree one invertible sheaf on $E$ such that $\mathcal{L}^{\sigma^{i}} \neq \mathcal{L}$ for $i=1,2,3$.

2. Since the curve $E$ has genus one, the Riemann-Roch Theorem and Serre duality give that, for all invertible sheaves $\mathcal{M}$ on $E$,

$$
h^{0}(\mathcal{M})-h^{1}(\mathcal{M})=\operatorname{deg}(\mathcal{M}) .
$$


In particular, if $\mathcal{M}$ is tame of positive degree, then $h^{0}(\mathcal{M})=\operatorname{deg}(\mathcal{M})$, and if $\mathcal{M}$ is tame of negative degree, then $h^{1}(\mathcal{M})=-\operatorname{deg}(\mathcal{M})$. The duality theorem combines with the fact that the canonical sheaf $\omega_{E}$ is isomorphic to the structure sheaf $\mathcal{O}_{E}$ to give that

$$
h^{0}(\mathcal{M})=h^{1}\left(\mathcal{M}^{-1}\right)
$$

for all invertible sheaves $\mathcal{M}$ on $E$.

Let $\mathcal{M}$ be an invertible sheaf on $E$ which is generated by its global sections. As in $[\mathrm{ATV} 1, \mathrm{p} .70]$, there is a canonical exact sequence

$$
0 \rightarrow \mathcal{M}^{\prime \prime} \rightarrow \mathcal{M}^{\prime} \rightarrow \mathcal{M} \rightarrow 0
$$

where $\mathcal{M}^{\prime}=\mathcal{O}_{E} \otimes H^{0}(\mathcal{M})$ and $\mathcal{M}^{\prime \prime}$ is the kernel of the canonical map $\mathcal{M}^{\prime} \rightarrow \mathcal{M}$. The sheaf $\mathcal{M}^{\prime}$ is a free $\mathcal{O}_{E}$-module of rank $h^{0}(\mathcal{M})$, and $\mathcal{M}^{\prime \prime}$ is locally free of rank $n=h^{0}(\mathcal{M})-1$. Also, $\bigwedge^{n} \mathcal{M}^{\prime \prime}=\mathcal{M}^{-1}$. We will make use of the following result, which is given in [ATV1, Proposition 7.17]:

Proposition 4.3 (Artin-Tate-Van den Bergh). Let $\mathcal{M}$ and $\mathcal{N}$ be invertible sheaves on $E$ such that $\mathcal{M}$ is generated by its global sections and $h^{1}(\mathcal{N})=0$. Let

$$
\mu=\mu_{\mathcal{M}, \mathcal{N}}: H^{0}(\mathcal{M}) \otimes H^{0}(\mathcal{N}) \rightarrow H^{0}(\mathcal{M} \otimes \mathcal{N})
$$

be the multiplication map. Then $\operatorname{ker} \mu \cong H^{0}\left(\mathcal{M}^{\prime \prime} \otimes \mathcal{N}\right)$, coker $\mu \cong H^{1}\left(\mathcal{M}^{\prime \prime} \otimes \mathcal{N}\right)$ and $H^{1}(\mathcal{M} \otimes \mathcal{N})=0$.

We are particularly interested in the sequence (4.2) when $\mathcal{M}$ is an invertible sheaf of degree two on $E$. In this case, we have that $n=1$, and so $\mathcal{M}^{\prime \prime}=\mathcal{M}^{-1}$.

Proof of Theorem 4.1(i)-(iv). The fact that $B_{0}=k$ is just the statement that $H^{0}\left(\mathcal{O}_{E}\right)=k$, which follows from [ATV1, Proposition 7.11]. Because the sheaves $\mathcal{L}_{n}$ are tame (Proposition 3.3), the Riemann-Roch Theorem gives that, for each $n$,

$$
\operatorname{dim}\left(B_{n}\right)=h^{0}\left(\mathcal{L}_{n}\right)=\operatorname{deg}\left(\mathcal{L}_{n}\right)=n .
$$

This proves Theorem 4.1(i).

The fact that $B$ is Noetherian follows from [AV, Theorem 1.4]. Suppose $u$ is a homogeneous element of $\operatorname{soc}\left(B_{B}\right)$. Then $u B_{2}=u H^{0}\left(\mathcal{L}_{2}\right)=0$. Since $\mathcal{L}_{2}$ is generated by global sections (Proposition 3.3), we have that $u=0$. The proof that $\operatorname{soc}\left({ }_{B} B\right)=0$ is similar.

Let $\mu_{2, n}: H^{0}\left(\mathcal{L}_{2}\right) \otimes H^{0}\left(\mathcal{L}_{n}^{\sigma^{2}}\right) \rightarrow H^{0}\left(\mathcal{L}_{n+2}\right)$ be the multiplication map for $n \geq 3$. Since $\mathcal{L}_{2}$ is generated by its sections, by the remarks following Proposition 4.3, we have that $\mathcal{L}_{2}^{\prime \prime}=\mathcal{L}_{2}^{-1}$. Also, $H^{1}\left(\mathcal{L}_{n}^{\sigma^{2}}\right)=0$, because this sheaf is tame and of positive degree. Now Proposition 4.3 gives that $\operatorname{coker}\left(\mu_{2, n}\right) \cong H^{1}\left(\mathcal{L}_{2}^{-1} \otimes \mathcal{L}_{n}^{\sigma^{2}}\right)$. But $\mathcal{L}_{2}^{-1} \otimes \mathcal{L}_{n}^{\sigma^{2}}$ is tame and of positive degree, and so its first cohomology vanishes. Hence, $\mu_{2, n}$ is surjective, which shows that $B_{2} B_{n}=B_{n+2}$.

If $\mathcal{L}_{2}^{-1} \otimes \mathcal{L}_{2}^{\sigma^{2}} ¥ \mathcal{O}_{E}$, the same proof shows that $B_{2} B_{2}=B_{4}$, completing the proof of (iii).

Part (iii) immediately gives that $B$ is generated as a $k$-algebra in degrees less than or equal to four. If $\mathcal{L}_{2}^{-1} \otimes \mathcal{L}_{2}^{\sigma^{2}} ¥ \mathcal{O}_{E}$, then part (iii) implies that $B$ is generated in degrees less than or equal to three.

Suppose that $\mathcal{L}_{2}^{-1} \otimes \mathcal{L}_{2}^{\sigma^{2}} \cong \mathcal{O}_{E}$. (It is easily checked that this occurs if and only if $E=E( \pm)$ or $E=E(a: b: 0)$.) We show that $B_{1} B_{3}+B_{3} B_{1}=B_{4}$. Proposition 3.4 gives that the maps $\mu_{1,3}$ and $\mu_{3,1}$ are injective. Thus, $B_{1} B_{3}$ and $B_{3} B_{1}$ are both three-dimensional subspaces of the four-dimensional vector space $B_{4}$. Suppose 
$B_{1} B_{3}=B_{3} B_{1}$. Let $\alpha \in k(E)$ be a nonzero element of $B_{1}$. Then $B_{1} B_{3}=\alpha H^{0}\left(\mathcal{L}_{3}^{\sigma}\right)$ and $B_{3} B_{1}=\alpha^{\sigma^{3}} H^{0}\left(\mathcal{L}_{3}\right)$. As $\mathcal{L}_{3}$ is generated by its global sections, $\mathcal{L}_{3} \cong \mathcal{L}_{3}^{\sigma}$. This reduces to $\mathcal{L} \cong \mathcal{L}^{\sigma^{3}}$, contradicting Lemma 3.5 .

By Proposition 3.4, the vector spaces $B_{2} B_{1}$ and $B_{1} B_{2}$ are both two-dimensional subspaces of $B_{3}$. If $B_{1} B_{2}+B_{2} B_{1} \neq B_{3}$, then $B_{2} B_{1}=B_{1} B_{2}$. As above, this implies that $\mathcal{L}_{2} \cong \mathcal{L}_{2}^{\sigma}$, which in turn implies that $\mathcal{L} \cong \mathcal{L}^{\sigma^{2}}$, a contradiction. Thus, $B$ is generated in degrees one and two.

As $\operatorname{dim} B_{1}=1$, only one generator of degree one is required. Since $\operatorname{dim} B_{2}=2$ and $\operatorname{dim} B_{1} B_{1}=1$, only one generator of degree two is required.

We can present $B$ as a graded quotient algebra of $T=k\{x, y\}$, where $\operatorname{deg}(x)=1$ and $\operatorname{deg}(y)=2$. Let $J \subset T=k\{x, y\}$ be a homogeneous ideal such that $T / J \cong B$ and let $\phi: T \rightarrow B$ be the graded algebra surjection. Complications arise at this point since the free algebra $T$ is not generated in degree one, and it is not true in general that $T_{i} T_{j}=T_{i+j}$. We use the notation of [ATV1]: for $l, m, n \geq 0$, define

$$
K_{l, m}=\operatorname{ker}\left(B_{l} \otimes B_{m} \rightarrow B_{l+m}\right),
$$

and let $\theta_{l, m}: T_{l} \otimes T_{m} \rightarrow B_{l} \otimes B_{m}$ be the surjective map induced by $\phi$.

We now proceed with the proof of Theorem 4.1(v).

Lemma 4.4. Preserve the hypothesis of Theorem 4.1(v) and the above notation. For $m \geq 2$,

$$
H^{0}\left(\mathcal{L}_{2}^{-1} \otimes \mathcal{L}_{m}^{\sigma^{2}}\right) \cong \frac{J_{m+2} \cap T_{2} T_{m}}{T_{2} J_{m}}
$$

Proof. The preimage of $K_{2, m}$ under $\theta_{2, m}$ can be identified with $J_{m+2} \cap T_{2} T_{m}$. The kernel of $\theta_{2, m}$ can be identified with $T_{2} J_{m}$. Hence $K_{2, m} \cong\left(J_{m+2} \cap T_{2} T_{m}\right) /\left(T_{2} J_{m}\right)$.

Set $\mathcal{M}=\mathcal{L}_{2}^{-1} \otimes \mathcal{L}_{m}^{\sigma^{2}}$. By Proposition 4.3,

$$
H^{0}(\mathcal{M}) \cong \operatorname{ker}\left(\mu_{2, m}: B_{2} \otimes B_{m} \rightarrow B_{m+2}\right) .
$$

Thus, $H^{0}(\mathcal{M}) \cong K_{2, m}$, proving the lemma.

For $m \geq 0$, let

and let

$$
M_{m, n}=H^{0}\left(\mathcal{L}_{2}^{-1} \otimes \mathcal{L}_{m}^{\sigma^{2}}\right) \otimes H^{0}\left(\mathcal{L}_{n}^{\sigma^{m+2}}\right)
$$

$$
N_{m}=H^{0}\left(\mathcal{L}_{2}^{-1} \otimes \mathcal{L}_{m}^{\sigma^{2}}\right)
$$

We will use $\rho_{m, n}$ to denote the sheaf multiplication $M_{n, m} \rightarrow N_{n+m}$.

Lemma 4.5. Under the hypotheses of Theorem 4.1(v), the function $\rho_{m, 2}$ is surjective if $m \geq 5$ and $m \neq 7$. If $E$ has at most three components, $\rho_{m, 2}$ is surjective for all $m \geq 5$.

Proof. This is a straightforward application of Proposition 4.3, setting $\mathcal{M}=\mathcal{L}_{2}^{\sigma^{m+2}}$ and $\mathcal{N}=\mathcal{L}_{2}^{-1} \otimes \mathcal{L}_{m}^{\sigma^{2}}$, and using the tameness of $\mathcal{N} \otimes \mathcal{M}^{-1}$ from Lemma 3.3.

Let $\rho_{m, n} \oplus \rho_{m-1, n+1}$ be the direct sum map $M_{m, n} \oplus M_{m-1, n+1} \rightarrow N_{m+n}$.

Lemma 4.6. Suppose $E$ has no more than three components. Then

(i) $\rho_{5,1} \oplus \rho_{3,3}$ is surjective,

(ii) $\rho_{4,1} \oplus \rho_{3,2}$ is surjective,

(iii) the cokernel of $\rho_{3,1}$ is one dimensional. 
Proof. By Proposition 3.4, the cokernel of $\rho_{5,1}$ is one dimensional. It is easily seen that this is also true of the cokernel of $\rho_{3,3}$. The image of $\rho_{3,3}$ is $\alpha H^{0}\left(\mathcal{L}_{3}^{\sigma^{5}}\right)$ for some nonzero rational function $\alpha$. This is a three dimensional subspace of the four dimensional space $N_{6}$. The image of $\rho_{5,1}$ inside of $N_{6}$ is a three dimensional subspace of the form $\beta H^{0}\left(\mathcal{L}_{2}^{-1} \otimes \mathcal{L}_{5}^{\sigma^{2}}\right)$ for some nonzero rational function $\beta$.

The only way the conclusion of (i) fails to hold is if the images of $\rho_{3,3}$ and $\rho_{5,1}$ are equal. If so, then $\alpha H^{0}\left(\mathcal{L}_{3}^{\sigma^{5}}\right)=\beta H^{0}\left(\mathcal{L}_{2}^{-1} \otimes \mathcal{L}_{5}^{\sigma^{2}}\right)$. The sheaves on either side of this equation are generated by their global sections by Proposition 3.4. Thus $\mathcal{L}_{3}^{\sigma^{5}} \cong \mathcal{L}_{2}^{-1} \otimes \mathcal{L}_{5}^{\sigma^{2}}$

Suppose that $\sigma$ is a translation on $E$ with respect to a group structure. Then the Theorem of the Square applied to the above isomorphism gives that $\mathcal{L} \cong \mathcal{L}^{\sigma}$, contradicting Lemma 3.5. If $\sigma$ is complex multiplication of order four on $E$, then the above isomorphism gives easily that $\mathcal{L} \cong \mathcal{L}^{\sigma}$, which again gives a contradiction. This proves (i).

The argument for (ii) is similar, and uses the fact that $\mathcal{L} ¥ \mathcal{L}^{\sigma^{2}}$.

By Proposition 3.4, $\rho_{3,1}$ is injective. By the Riemann-Roch Theorem, $\operatorname{dim}\left(M_{3,1}\right)$ $=1$ and $\operatorname{dim}\left(N_{4}\right)=2$. This proves (iii).

Lemma 4.7. Preserve the hypothesis of Theorem 4.1(v).

(i) Suppose $\mathcal{L}_{2}^{-1} \otimes \mathcal{L}_{2}^{\sigma^{2}} \neq \mathcal{O}_{E}$. Then for $m \geq 4$,

$$
T_{m+2}=T_{2} T_{m}+J_{5} T_{m-3}+J_{4} T_{m-2}=T_{m} T_{2}+T_{m-3} J_{5}+T_{m-2} J_{4} .
$$

(ii) Suppose $\mathcal{L}_{2}^{-1} \otimes \mathcal{L}_{2}^{\sigma^{2}} \cong \mathcal{O}_{E}$. Then for $m>4$,

$$
T_{m+2}=T_{2} T_{m}+J_{5} T_{m-3}+J_{6} T_{m-4}=T_{m} T_{2}+T_{m-3} J_{5}+T_{m-4} J_{6},
$$

and

$$
\operatorname{dim}_{k} \frac{T_{6}}{T_{2} T_{4}+J_{5} T_{1}} \leq 1 .
$$

Proof. By Theorem 4.1(iii), we have that $B_{2} B_{m}=B_{m+2}$ for $m \geq 2$. Therefore, $T_{4}=T_{2} T_{2}+J_{4}$ and $T_{5}=T_{2} T_{3}+J_{5}$. Let $m \geq 4$ and let $u$ be any monomial in $T_{m+2}$ but not in $T_{2} T_{m}$. Then $u$ must begin with $x y$, and so either $u=x y x t$ for some $t \in T_{m-2}$, or $u=x y^{2} t$ for some $t \in T_{m-3}$. But

$$
x y x t \in T_{4} T_{m-2}=T_{2} T_{2} T_{m-2}+J_{4} T_{m-2} \subseteq T_{2} T_{m}+J_{4} T_{m-2},
$$

and

$$
x y^{2} t \in T_{5} T_{m-3}=T_{2} T_{3} T_{m-3}+J_{5} T_{m-3} \subseteq T_{2} T_{m}+J_{5} T_{m-3},
$$

proving the first part of (i). The second equality in (i) is proven by a symmetric argument.

Theorem 4.1(iii) gives that $T_{5}=T_{2} T_{3}+J_{5}$ and $T_{6}=T_{2} T_{4}+J_{6}$. For $m>4$, the proof proceeds as above, and so we will omit it. Let $u$ be any monomial of $T_{6}$ which is not in $T_{2} T_{4}$. Again, $u$ must start with $x y$, and so $u$ is one of $x y^{2} x, x y x^{3}$ and $x y x y$. As above, we have $x y^{2} x, x y x^{3} \in T_{2} T_{4}+J_{5} T_{1}$, proving the second part of (ii).

Lemma 4.8. Under the hypothesis of Theorem 4.1(v),

$$
T_{2} T_{6}+J_{5} T_{3}+T_{3} J_{5}=T_{6} T_{2}+J_{5} T_{3}+T_{3} J_{5} .
$$


Proof. By Theorem 4.1(iii), $T_{5}=T_{2} T_{3}+J_{5}=T_{3} T_{2}+J_{5}$. We show one containment:

$$
\begin{aligned}
T_{2} T_{6} & \subseteq T_{6} T_{2}+T_{2} T_{3} T_{3} \\
& \subseteq T_{6} T_{2}+T_{5} T_{3} \\
& \subseteq T_{6} T_{2}+T_{3} T_{2} T_{3}+J_{5} T_{3} \\
& \subseteq T_{6} T_{2}+T_{3} T_{3} T_{2}+J_{5} T_{3}+T_{3} J_{5} \\
& =T_{6} T_{2}+J_{5} T_{3}+T_{3} J_{5} .
\end{aligned}
$$

The proof of the reverse containment is symmetric, and this proves the lemma.

Lemma 4.9. Preserve the hypothesis of Theorem 4.1(v). Then for $m>4$,

$$
J_{m+4}=J_{m+2} T_{2}+T_{2} J_{m+2}+J_{5} T_{m-1}+J_{6} T_{m-2} .
$$

If $E$ has at most three components, then

(i) $J_{8}=J_{7} T_{1}+J_{5} T_{3}+T_{3} J_{5}+T_{2} J_{6}=T_{1} J_{7}+T_{3} J_{5}+J_{5} T_{3}+J_{6} T_{2}$,

(ii) $J_{7}=J_{6} T_{1}+J_{5} T_{2}+T_{2} J_{5}=T_{1} J_{6}+T_{2} J_{5}+J_{5} T_{2}$,

(iii) $J_{6}=J_{5} T_{1}+J_{4} T_{2}+T_{2} J_{4}+V$, where $V$ is a vector space of dimension at most one.

Proof. The fact that $\rho_{m, 2}$ is surjective (Lemma 4.5) combines with Lemma 4.4 to give a surjective map

$$
\frac{J_{m+2} \cap T_{2} T_{m}}{T_{2} J_{m}} \otimes T_{2} \rightarrow \frac{J_{m+4} \cap T_{2} T_{m+2}}{T_{2} J_{m+2}},
$$

which is induced by multiplication in $T$. This implies that

$$
J_{m+2} T_{2}+T_{2} J_{m+2} \supseteq J_{m+4} \cap T_{2} T_{m+2} .
$$

Now

$$
\begin{aligned}
J_{m+4} & =J_{m+4} \cap T_{m+4} \\
& =J_{m+4} \cap\left(T_{2} T_{m+2}+J_{5} T_{m-1}+J_{6} T_{m-2}\right) \quad(\text { Lemma } 4.7) \\
& =\left(J_{m+4} \cap T_{2} T_{m+2}\right)+J_{5} T_{m-1}+J_{6} T_{m-2} \\
& \subseteq J_{m+2} T_{2}+T_{2} J_{m+2}+J_{5} T_{m-1}+J_{6} T_{m-2} .
\end{aligned}
$$

The fact that $\rho_{5,1} \oplus \rho_{3,3}$ is surjective (Lemma 4.6) combines with Lemma 4.4 to give a surjective map

$$
\left(\frac{J_{7} \cap T_{2} T_{5}}{T_{2} J_{5}} \otimes T_{1}\right) \oplus\left(\left(J_{5} \cap T_{2} T_{3}\right) \otimes T_{3}\right) \rightarrow \frac{J_{8} \cap T_{2} T_{6}}{T_{2} J_{6}},
$$

where all of the maps are induced by multiplication and addition in $T$. This implies

$$
J_{7} T_{1}+J_{5} T_{3}+T_{2} J_{6} \supseteq J_{8} \cap T_{2} T_{6} .
$$

Now

$$
\begin{aligned}
J_{8} & =J_{8} \cap T_{8} \\
& =J_{8} \cap\left(T_{6} T_{2}+T_{3} J_{5}+T_{2} J_{6}\right) \quad(\text { Lemma } 4.7) \\
& =J_{8} \cap\left(T_{2} T_{6}+J_{5} T_{3}+T_{3} J_{5}+T_{2} J_{6}\right) \quad(\text { Lemma 4.8) } \\
& =\left(J_{8} \cap T_{6} T_{2}\right)+J_{5} T_{3}+T_{3} J_{5}+T_{2} J_{6} \\
& \subseteq J_{7} T_{1}+J_{5} T_{3}+T_{3} J_{5}+T_{2} J_{6},
\end{aligned}
$$

proving the first half of (i). The second half of (i) is symmetric.

Similarly, the fact that $\rho_{4,1} \oplus \rho_{3,2}$ is surjective (Lemma 4.6) gives that

$$
J_{6} T_{1}+J_{5} T_{2}+T_{2} J_{5} \supseteq J_{7} \cap T_{2} T_{5} .
$$


Then

$$
\begin{aligned}
J_{7} & =J_{7} \cap T_{7} \\
& =J_{7} \cap\left(T_{2} T_{5}+J_{5} T_{2}+J_{6} T_{1}\right) \quad \text { (Lemma 4.7) } \\
& \subseteq J_{6} T_{1}+J_{5} T_{2}+T_{2} J_{5},
\end{aligned}
$$

proving the first half of (ii).

Suppose $\mathcal{L}_{2} \otimes \mathcal{L}_{2}^{\sigma^{2}} ¥ \mathcal{O}_{E}$. The fact that $\rho_{3,1}$ has a one dimensional cokernel (Lemma 4.6) shows that there exists a one dimensional subspace $V$ of $T_{6}$ such that

$$
J_{5} T_{1}+T_{2} J_{4}+V \supseteq J_{6} \cap T_{2} T_{4} .
$$

Then

$$
\begin{aligned}
J_{6} & =J_{6} \cap\left(T_{2} T_{4}+J_{5} T_{1}+J_{4} T_{2}\right) \\
& =\left(J_{6} \cap T_{2} T_{4}\right)+J_{5} T_{1}+J_{4} T_{2} \\
& \subseteq J_{5} T_{1}+T_{2} J_{4}+J_{4} T_{2}+V .
\end{aligned}
$$

Suppose $\mathcal{L}_{2} \otimes \mathcal{L}_{2}^{\sigma^{2}} \cong \mathcal{O}_{E}$. The fact that $\rho_{2,2}$ is surjective gives that

$$
T_{2} J_{4}+J_{4} T_{2} \supseteq J_{6} \cap T_{2} T_{4} .
$$

Lemma 4.7 shows that there exists a subspace $V$ of $T_{6}$ of dimension at most one, such that $T_{6}=T_{2} T_{4}+J_{4} T_{2}+J_{5} T_{1}+V$. Now

$$
\begin{aligned}
J_{6} & =J_{6} \cap\left(T_{2} T_{4}+J_{4} T_{2}+J_{5} T_{1}+V\right) \\
& =\left(J_{6} \cap T_{2} T_{4}\right)+J_{4} T_{2}+J_{5} T_{1}+V \\
& =T_{2} J_{4}+J_{4} T_{2}+J_{5} T_{1}+V,
\end{aligned}
$$

and this completes the proof of (iv).

Now we complete the proof of Theorem $4.1(\mathrm{v})$ in the case that $E$ has at most three components. The fact that $\operatorname{dim} T_{4}-\operatorname{dim} B_{4}=5-4=1$ implies that $J_{4}$ is one dimensional. Let $j_{4}$ be a nonzero element of $J_{4}$. It is easy to see that $x j_{4}$ and $j_{4} x$ are linearly independent elements of $J_{5}$. This, combined with the fact that $\operatorname{dim} J_{5}=3$, shows that we need exactly one relation of degree 5 , which we will call $j_{5}$. Lemma 4.9 shows that we need at most one new relation of degree six, and no new relations in any higher degrees. Call the degree six relation $j_{6}$ (which could at this point be generated by $j_{4}$ and $\left.j_{5}\right)$. Now $B \cong T /\left(j_{4}, j_{5}, j_{6}\right)$, as desired.

To complete the proof in the six component case, we require a result to replace Lemma 4.5 when $m=7$.

Lemma 4.10. $J_{11} \subseteq J_{6} T_{5}+T_{2} J_{9}+J_{5} T_{6}+J_{4} T_{7}$

Proof. Consider the multiplication map

$$
\mu: H^{0}\left(\mathcal{L}_{2}^{-1} \otimes \mathcal{L}_{4}^{\sigma^{2}}\right) \otimes H^{0}\left(\mathcal{L}_{5}^{\sigma^{6}}\right) \rightarrow H^{0}\left(\mathcal{L}_{2}^{-1} \otimes \mathcal{L}_{9}^{\sigma^{2}}\right)
$$

By [ATV1, Proposition 7.17], the cokernel of this map is isomorphic to $H^{1}(\mathcal{M})$, where $\mathcal{M}=\mathcal{L}_{2} \otimes\left(\mathcal{L}_{4}^{\sigma^{2}}\right)^{-1} \otimes \mathcal{L}_{5}^{\sigma^{6}}$. The sheaf $\mathcal{M}$ is of positive degree, and is tame by [ATV1, Proposition 7.10]. Thus, $\mu$ is surjective.

This implies that

is surjective. Thus,

$$
\frac{J_{6} \cap T_{2} T_{4}}{T_{2} J_{4}} \otimes B_{5} \rightarrow \frac{J_{11} \cap T_{2} T_{9}}{T_{2} J_{9}}
$$

$$
\left(J_{6} \cap T_{2} T_{4}\right) T_{5}+T_{2} J_{9}=J_{11} \cap T_{2} T_{9}
$$


By Lemma 4.6, $T_{11}=T_{2} T_{9}+J_{5} T_{6}+J_{4} T_{7}$. Now

$$
\begin{aligned}
J_{11} & =J_{11} \cap T_{11}=J_{11} \cap\left(T_{2} T_{9}+J_{5} T_{6}+J_{4} T_{7}\right) \\
& \left.=\left(J_{11} \cap T_{2} T_{9}\right)+J_{5} T_{6}+J_{4} T_{7}\right) \\
& =J_{6} T_{5}+T_{2} J_{9}+J_{5} T_{6}+J_{4} T_{7},
\end{aligned}
$$

which gives the result.

Now the ideal $J \subset T$ of defining relations of $B$ is generated in degrees less than or equal to eight. It is clear that $x^{6} \in J$. It is also easy to show that the defining relations of $A$ are in $J$. (This fact will be used in the next section to provide a surjective homomorphism $h: A(\mathbf{a}) \rightarrow B$ ). Also, from Proposition 4.9, we know that $J$ is generated as an ideal of $T$ in degrees eight and lower. We show that the relations $\left\{f_{1}, f_{2}\right\}$ coming from $A$ together with the relation $x^{6}=0$ are enough to assure that $B$ has the right Hilbert series in degrees lower than 8 , and therefore these relations generate all of $J$.

Let $Q$ be the ideal of $T$ generated by $\left\{f_{1}, f_{2}, x^{6}\right\}$. Brute force computation of bases for $Q_{6}, Q_{7}$ and $Q_{8}$ can be done by hand, and we find that $\operatorname{dim} Q_{6}=7$, $\operatorname{dim} Q_{7}=14$, and $\operatorname{dim} Q_{8}=26$. This shows that $\operatorname{dim}(T / Q)_{6}=7, \operatorname{dim}(T / Q)_{7}=8$ and $\operatorname{dim}(T / Q)_{8}=10$. This gives that $(T / Q)_{n}=B_{n}$ for $n \leq 8$. Thus, $Q$ and $J$ agree up through degree 8 , and so Proposition 4.9 gives that $Q=J$.

\section{The Main Theorem}

In this section, we prove the regularity of the algebras $A( \pm)$ and the algebras $A(\mathbf{a})$ such that $\mathbf{a} \notin S$. First we give the precise definition.

Let $A$ be a connected graded $k$ algebra. The module $A / \bigoplus_{i>0} A_{i}$ will be denoted by ${ }_{A} k$ or $k_{A}$ depending on whether we are considering it as a left or right $A$-module.

Definition 5.1. A connected $\mathbb{N}$-graded $k$-algebra $A$ is said to be Artin-Schelter regular, or AS-regular, if:

(i) $A$ has finite global dimension $m$,

(ii) $A$ has polynomial growth, that is, there exist $c \in \mathbb{R}^{+}$and $r \in \mathbb{N}$ such that for all $n \in \mathbb{N}$ we have $\operatorname{dim}\left(A_{n}\right) \leq c n^{r}$, and

(iii) $A$ is Gorenstein, that is, the projective modules appearing in a minimal projective resolution $\mathbf{P}$ of the graded module ${ }_{A} k$ are finitely generated, and the dual complex $\mathbf{P}^{*}$ is a projective resolution of a shift of the graded right module $k_{A}$.

We first dispense with some degenerate cases.

Proposition 5.2. $A(\mathbf{a})$ is not $A S$-regular if $\mathbf{a} \in S$.

Proof. In [St2] it is shown that, if $A$ is AS-regular of global dimension three and is generated by two elements, one in degree one and one in degree two, then $A$ must have the same Hilbert series as the commutative polynomial ring $k\left[X_{1}, X_{2}, X_{3}\right]$ graded so that $\operatorname{deg}\left(X_{i}\right)=i$. Brute force computation using Schelter's AFFINE program shows that this is not the case when $\mathbf{a} \in S$.

For the rest of this section, we will fix the following notation: let $A$ represent $A( \pm)$ or $A(\mathbf{a})$ such that $\mathbf{a} \notin S$ and $b, a+c \neq 0$. We will use the symbols $x$ and $y$ both for the generators of $T=k\{x, y\}$ and for their images in $A$. Let $(E, \sigma, \mathcal{L})$ be the geometric data associated to $A$, and set $B=B(E, \sigma, \mathcal{L})$. 
We will work in the category of $\mathbb{Z}$-graded $A$-modules. We use $\operatorname{Hom}_{A}^{d}(M, N)$ to denote the set of graded module homomorphisms from $M$ to $N$ of degree $d$. We set $\underline{\operatorname{Hom}}_{A}(M, N)=\bigoplus_{d \in \mathbb{Z}} \operatorname{Hom}_{A}^{d}(M, N)$, and denote the corresponding derived functors by $\underline{\operatorname{Ext}}_{A}^{i}(M, N)$.

Proposition 5.3. Under the above hypotheses, there is a surjective homomorphism of graded $k$-algebras $h: A \rightarrow B$ sending $x$ to $x_{0}$ and $y$ to $y_{0}$.

Proof. As in section 3, we may regard $x_{0} \in B_{1}$ and $y_{0} \in B_{2}$. Define a graded algebra homomorphism $\phi: T \rightarrow B$ by $x \mapsto x_{0}$ and $y \mapsto y_{0}$. As $B$ is generated in degrees one and two by Lemma $4.1(\mathrm{iv}), \phi$ is surjective. We claim that this $\phi$ factors through $A$. To show this, it is sufficient to show that $\phi\left(f_{1}\right)=\phi\left(f_{2}\right)=0$. But $\phi\left(f_{1}\right)=\phi\left(f_{2}\right)=0$ are exactly the relations (2.1.2) and (2.1.1) (or (2.4.2) and (2.4.1)) which define $G_{5}$, and thus they act as zero on $E$. So the map $\phi$ factors through $A$ to give the required surjective graded homomorphism $h: A \rightarrow B$.

Now we examine the kernel of the map $h$.

Proposition 5.4. The kernel of $h: A \rightarrow B$ is generated by one nonzero normal element $d \in A_{6}$.

Proof. In the six component case, it is obvious that $x^{6}$ is a central element of $\operatorname{ker}(h)$. By Theorem $4.1, A / x^{6} A \cong B$.

Assume that $E$ has at most three components. Let $\tau: T \rightarrow A$ be the obvious map. Let $I=\operatorname{ker} \tau$, and let $J=\operatorname{ker}(\phi: T \rightarrow B)$. Let $K=\operatorname{ker}(h)=I / J$. It is clear that $\operatorname{dim}\left(A_{i}\right)=\operatorname{dim}\left(B_{i}\right)$ for $i \leq 5$, and so $K_{i}=0$. Lemma 4.9 gives that, for $m>4$,

$$
J_{m+4}=J_{m+2} T_{2}+T_{2} J_{m+2}+J_{5} T_{m-1}+J_{6} T_{m-2} .
$$

Application of $\tau$ to this equation gives that

$$
K_{m+4}=K_{m+2} A_{2}+A_{2} K_{m+2}+K_{6} A_{m-2} .
$$

This shows that the kernel of $h$ is generated in degrees eight and lower.

If $E$ has no more than three components, we apply $\tau$ to each of the equations in Lemma 4.9(ii)-(iv), and obtain

$$
\begin{aligned}
& K_{8}=K_{7} A_{1}+A_{2} K_{6}=A_{1} K_{7}+K_{6} A_{2}, \\
& K_{7}=K_{6} A_{1}=A_{1} K_{6}, \\
& K_{6}=V /(V \cap I) .
\end{aligned}
$$

This shows that $K$ is generated by $K_{6}$, and that $\operatorname{dim}\left(K_{6}\right) \leq 1$.

It is easily checked from the relations defining $A$ that $\operatorname{dim}\left(I_{6}\right) \leq 6$, and it is known that $\operatorname{dim}\left(J_{6}\right)=\operatorname{dim}\left(T_{6}\right)-\operatorname{dim}\left(I_{6}\right)=7$. Therefore, $\operatorname{dim}\left(K_{6}\right) \geq 1$, and so $\operatorname{dim}\left(K_{6}\right)=1$. Let $d$ be a basis for $K_{6}$. Then $K_{7}=d A_{1}=A_{1} d$. Also, $K_{8}=$ $A_{1} d A_{1}+A_{2} d=A_{2} d$ and, similarly, $K_{8}=d A_{2}$. This shows that $K$ is generated by a single nonzero element of degree six, and moreover that $A_{1} d=d A_{1}$ and $A_{2} d=d A_{2}$. As $A$ is generated in degrees one and two, this shows that $d$ is a normal element of A.

Remark. An explicit expression for the element $d$ can be found using Schelter's AFFINE program. This is given in [St1].

Define a $2 \times 2$ matrix $M=\left(m_{i j}\right)$ with homogeneous entries in $T$ by

$$
M\left(\begin{array}{l}
x \\
y
\end{array}\right)=\left(\begin{array}{l}
f_{1} \\
f_{2}
\end{array}\right) \text {. }
$$


Consider the following sequence of graded left $A$-modules:

$$
0 \rightarrow A[-6] \stackrel{(x, y)}{\longrightarrow} A[-5] \oplus A[-4] \stackrel{M}{\longrightarrow} A[-1] \oplus A[-2] \stackrel{\left(\begin{array}{l}
x \\
y
\end{array}\right)}{\longrightarrow} A \stackrel{p r}{\longrightarrow}_{A} k \rightarrow 0 .
$$

The maps in the sequence (5.5) are defined as follows:

$$
\begin{aligned}
& (x, y): u \mapsto(u x, u y), \\
& M:(u, v) \mapsto\left(u m_{11}+v m_{21}, u m_{21}+v m_{22}\right), \\
& \left(\begin{array}{c}
x \\
y
\end{array}\right):(u, v) \mapsto u x+v y, \\
& p r: \text { the projection } A \rightarrow A / A_{+} \cong_{A} k,
\end{aligned}
$$

where $A_{+}=\bigoplus_{i>0} A_{i}$.

Our goal is to show that (5.5) is a free resolution of the module ${ }_{A} k$. We begin with the following lemma.

Lemma 5.6. The sequence (5.5) is a complex, and is exact at each of the first three terms from the right.

Proof. The fact that (5.5) is exact at the ${ }_{A} k$ term is evident from the definition of the map $p r$. Also, (5.5) is exact at the terms $A$ and $A[-1] \oplus A[-2]$ by [W, Lemmas $7,8]$.

Now we need only verify that $\operatorname{im}(x, y) \subseteq \operatorname{ker}(M)$. If $A=A(1: b: c)$, then $(x, y) M=\left(f_{1}, f_{2}\right)$, showing that $(x, y) \in \operatorname{ker}(M)$. It follows immediately that $A(x, y) \subset \operatorname{ker}(M)$. If $A=A( \pm)$, then $(x, y) M=\left( \pm i f_{1}+2 f_{2} x, f_{2}\right)$. This shows that $(x, y) \in \operatorname{ker}(M)$, and hence $A(x, y) \subset \operatorname{ker}(M)$.

Theorem 5.7. Under the above assumptions on $A$, the sequence (5.5) is exact and $d$ is a regular element of $A$. Furthermore, $A$ has the same Hilbert series as the commutative polynomial ring $k\left[X_{1}, X_{2}, X_{3}\right]$ graded so that $\operatorname{deg}\left(x_{i}\right)=i$.

Proof. Let $Z$ be the right annihilator of $d$. Let $F=k\left[X_{1}, X_{2}, X_{3}\right]$ be the polynomial ring, graded so that $\operatorname{deg}\left(X_{i}\right)=i$. For each $n \in \mathbb{Z}$, set

$$
\begin{aligned}
& a_{n}=\operatorname{dim} A_{n}, \\
& z_{n}=\operatorname{dim} Z_{n}, \\
& f_{n}=\operatorname{dim} F_{n}, \\
& e_{n}=a_{n}-f_{n} .
\end{aligned}
$$

Note that $a_{n}=z_{n}=f_{n}=e_{n}=0$ for all $n<0$. If $\theta: N \rightarrow N^{\prime}$ is a graded homomorphism of graded $A$-modules, let $\theta^{n}: N_{n} \rightarrow N_{n}^{\prime}$ be the $n^{\text {th }}$ graded part of $\theta$. We examine the graded pieces of sequence (5.5):

$$
0 \rightarrow A_{n-6} \stackrel{(x, y)}{\longrightarrow} A_{n-5} \oplus A_{n-4} \stackrel{M^{n}}{\longrightarrow} A_{n-1} \oplus A_{n-2} \stackrel{\left(\begin{array}{l}
x \\
y
\end{array}\right)^{n}}{\longrightarrow} A \stackrel{p r^{n}}{\longrightarrow}\left({ }_{A} k\right)_{n} \rightarrow 0 .
$$

By Lemma 5.6, (5.5) is exact at the first three terms from the right, and

$$
(x, y)^{n} \subseteq \operatorname{ker}\left(M^{n}\right) .
$$

Let

$$
P_{n}=\operatorname{ker}(x, y)^{n}
$$


and

$$
Q_{n}=\frac{\operatorname{ker}\left(M^{n}\right)}{\operatorname{im}(x, y)^{n}} .
$$

Note that $P_{n}$ and $Q_{n}$ are the only possible nonzero homology groups of (5.8). Let $p_{n}=\operatorname{dim} P_{n}$ and $q_{n}=\operatorname{dim} Q_{n}$. From the sequence (5.8), for all $n \in \mathbb{Z}$ we have

$$
a_{n-6}-a_{n-5}-a_{n-4}+a_{n-2}+a_{n-1}-a_{n}+\delta_{n, 0}=p_{n}-q_{n} .
$$

For the polynomial ring, we have

$$
f_{n-6}-f_{n-5}-f_{n-4}+f_{n-2}+f_{n-1}-f_{n}+\delta_{n, 0}=0
$$

for all $n \in \mathbb{Z}$. This gives that

$$
e_{n-6}-e_{n-5}-e_{n-4}+e_{n-2}+e_{n-1}-e_{n}=p_{n}-q_{n}
$$

for all $n \in \mathbb{Z}$.

We have an exact sequence of right modules

$$
0 \rightarrow Z \stackrel{i}{\longrightarrow} A \stackrel{d \cdot}{\longrightarrow} A \stackrel{h}{\longrightarrow} B \rightarrow 0,
$$

whose graded pieces are

$$
0 \rightarrow Z_{n-6} \stackrel{i^{n}}{\longrightarrow} A_{n-6} \stackrel{d .}{\longrightarrow} A_{n} \stackrel{h^{n}}{\longrightarrow} B_{n} \rightarrow 0 .
$$

By Theorem 4.1(i), we have that $\operatorname{dim} B_{n}=n$ for all $n \geq 1$, and $\operatorname{dim} B_{0}=1$. Thus for $n \geq 0$,

$$
z_{n-6}-a_{n-6}+a_{n}-n-\delta_{n, 0}=0 .
$$

By [Ha, Exercise IV.4.1], we have that $B(E, 1, \mathcal{L}) \cong F / w F$ for appropriate $w \in F_{6}$. Thus, for $n \geq 0$ we have

$$
f_{n}-f_{n-6}-n-\delta_{n, 0}=0
$$

and so

$$
z_{n-6}=e_{n-6}-e_{n}
$$

for all $n \geq 0$. Also, equation (5.10) holds trivially for $n<0$. As $z_{n-6}$ is always nonnegative, for all $n$ we have $e_{n} \leq e_{n-6}$. This, combined with the fact that $e_{i}=0$ for $i<0$, gives that $e_{n} \leq 0$ for all $n$.

Let $H(m)$ be the statement

$$
e_{m}=p_{m}=q_{m}=z_{m-6}=0 .
$$

We prove that $H(m)$ is true by induction. Notice that $H(m)$ is trivially true for $m<0$. Suppose, for some $m \in \mathbb{Z}$, that $H(n)$ is true for all $n<m$. Then $e_{n}=0$ for all $n<m$, which gives

$$
-e_{m}=p_{m}-q_{m}=z_{m-6} .
$$

Let $a \in A_{m-6}$ be any element such that $(a x, a y)=(0,0)$. Taking images in $B$, we obtain that $h(a) h(x)=0$ and $h(a) h(y)=0$, showing that $h(a) \in \operatorname{soc}\left(B_{B}\right)$. Thus, $h(a)=0$ by Theorem 4.1(ii). By Lemma 5.4, this gives $a \in d A$. Suppose $a=d t$ for some $t \in A_{m-12}$. Then $d t x=d t y=0$ implies that $t x \in Z_{m-11}$ and $t y \in Z_{m-10}$. By the induction hypothesis, $Z_{m-11}=Z_{m-10}=0$, and so $t x=t y=0$. But this gives that $t \in P_{m-6}$, which is zero by the induction hypothesis. Hence, $t=0$, and so $a=0$. This shows that $P_{m}=0$.

So therefore $p_{m}=0$. This gives that $e_{m}=q_{m}$. But $e_{m} \leq 0$ and $q_{m} \geq 0$, and so $e_{m}=q_{m}=0$. Finally, $z_{m-6}=-e_{m}=0$. So $H(m)$ holds, and the induction is complete. 
Now we have that $p_{n}=q_{n}=0$ for all $n$. This shows that sequence (5.5) is exact. Also, $z_{n}=0$ for all $n$, and so the right annihilator of $d$ is zero. This shows that $d$ is a right regular element of $A$.

As $d$ is a normal element of $A$, for all $n \in \mathbb{N}$ we have $A_{n} d=d A_{n}$. Also, as $d$ is right regular, $\operatorname{dim} d A_{n}=\operatorname{dim} A_{n}$. This gives that $\operatorname{dim} A_{n} d=\operatorname{dim} A_{n}$ for all $n \in \mathbb{N}$, and so $d$ is a left regular element of $A$.

This gives:

Corollary 5.11. Let $A$ represent $A( \pm)$ or $A(\mathbf{a})$ such that $\mathbf{a} \notin S$. Then $A$ is a Noetherian domain.

Proof. By Lemma 5.4 and Theorem 5.7, the element $d \in A_{6}$ is a homogeneous regular normal element of positive degree. By Theorem 4.1(ii), the factor ring $B=A / d A$ is Noetherian. Now [L, Theorem 3.5] gives that $A$ is Noetherian.

The fact that $A$ is a domain is now given in [Sn].

The following easy lemma is a result of the symmetric nature of the relations defining $A( \pm)$ and $A(\mathbf{a})$.

Lemma 5.12. (i) If a $\notin S$, then there exists a graded algebra isomorphism $g$ : $A(\mathbf{a}) \rightarrow A(\mathbf{a})^{\text {op }}$ such that $g(x)=x$ and $g(y)=y$.

(ii) There is a graded algebra isomorphism $g: A( \pm) \rightarrow A(\mp)^{\text {op }}$ such that $g(x)=x$ and $g(y)=y$.

Now we come to our main result.

Theorem 5.13. Let $A$ represent $A( \pm)$ or $A(\mathbf{a})$ such that $\mathbf{a} \notin S$. Then $A$ is $A S$ regular of global dimension three.

Proof. We begin by applying $\underline{\operatorname{Hom}}_{A}(-, A)$ to the sequence (5.5) of graded left $A$ modules, which is exact by Theorem 5.7. The result is the sequence of graded right A-modules

$$
0 \rightarrow A \stackrel{\gamma}{\longrightarrow} A[1] \oplus A[2] \stackrel{\beta}{\longrightarrow} A[5] \oplus A[4] \stackrel{\alpha}{\longrightarrow} A[6] \rightarrow 0,
$$

where the maps are given by:

$$
\begin{aligned}
& \gamma: u \mapsto(x u, y u), \\
& \beta:(u, v) \mapsto\left(m_{11} u+m_{12} v, m_{21} u+m_{22} v\right), \\
& \alpha:(u, v) \mapsto x u+y v, \\
& p r: A \rightarrow A / A_{+} \cong k_{A} .
\end{aligned}
$$

We shift the grading on the sequence (5.14) by six to obtain the sequence

$$
0 \rightarrow A[-6] \stackrel{\gamma}{\longrightarrow} A[-5] \oplus A[-4] \stackrel{\beta}{\longrightarrow} A[-1] \oplus A[-2] \stackrel{\alpha}{\longrightarrow} A \rightarrow 0
$$

and the form for the maps listed above does not change. We may consider sequence (5.15) as a sequence of graded left $A^{o p}$-modules. By Lemma 5.12, $A^{o p}$ is isomorphic to $A$ in the case that $A=A(\mathbf{a})$ and to $A(\mp)$ in the case that $A$ is $A( \pm)$. If we append ${ }_{A} k$ to the end of (5.15) using the projection $A \rightarrow A / A_{+}$, then we obtain the sequence

$$
0 \rightarrow A[-6] \stackrel{\gamma}{\longrightarrow} A[-5] \oplus A[-4] \stackrel{\beta}{\longrightarrow} A[-1] \oplus A[-2] \stackrel{\alpha}{\longrightarrow} A \rightarrow_{A} k \rightarrow 0 .
$$


This is exactly (5.5) for $A^{o p}$, which is exact by Theorem 5.7.

This shows that (5.15) is exact except at the right-hand end, where the homology module is the one dimensional graded right $A$-module $k_{A}$. This shows that

$$
\begin{aligned}
& \underline{\operatorname{Ext}}_{A}^{3}{ }_{A}\left({ }_{A} k, A\right) \cong k_{A}[6], \\
& \underline{\operatorname{Ext}}_{A}^{i}\left({ }_{A} k, A\right)=0 \text { if } i \neq 3 .
\end{aligned}
$$

The exactness of (5.5) shows that $\operatorname{gldim} A \leq 3$ by [CE, Theorem VIII.6.2 and Corollary VIII.6.4]. As $\underline{\operatorname{Ext}}_{A}^{3}\left({ }_{A} k, A\right)$ is nonzero, we must have gldim $A=3$. Hence, the resolution (5.5) is a minimal resolution of ${ }_{A} k$. The condition on the Ext groups above, together with the fact that the resolution (5.5) contains only finitely generated modules, shows that $A$ is Gorenstein. The fact that $A$ has polynomial growth follows from the fact that $A$ has the Hilbert series of a polynomial ring in three variables, which is given by Theorem 5.7. Hence, $A$ is AS-regular of global dimension three.

\section{ACKNOWLEDGMENT}

A large portion of this work was completed while the author was a $\mathrm{PhD}$ student at the University of Michigan under the supervision of J. T. Stafford. The author wishes to thank Professor Stafford for his guidance and support. The author also wishes to thank S. P. Smith for helpful conversations on this subject.

A portion of this work was completed while the author was visiting the University of Wales, and the author wishes to thank the mathematics faculty at Swansea and Aberystwyth for their hospitality and financial support.

\section{REFERENCES}

[A] M. Artin, Geometry of quantum planes, in Azumaya Algebras, Actions and Groups (Eds. D. Haile and J. Osterberg), Contemp. Math., Vol. 124, Amer. Math. Soc., Providence, 1992, pp. 1-15. MR 93b:14004

[ASch] M. Artin and W. Schelter, Graded algebras of global dimension 3, Adv. Math., 66 (1987), 171-216. MR 88k:16003

[ASt] M. Artin and J. T. Stafford, Noncommutative graded domains with quadratic growth, Inventiones Math., 122 (1995), 231-276. MR 96g:16027

[ATV1] M. Artin, J. Tate and M. Van den Bergh, Some algebras associated to automorphisms of curves, in The Grothendieck Festschrift (Eds. P. Cartier, et al.), Birkhäuser, Basel, 1990, pp. 31-85. MR 92e: 14002

[ATV2] M. Artin, J. Tate and M. Van den Bergh, Modules over regular algebras of dimension 3, Inventiones Math., 106 (1991), 335-389. MR 93e:16055

[AV] M. Artin and M. Van den Bergh, Twisted homogeneous coordinate rings, J. Algebra, 133 (1990), 249-271. MR 91k:14003

[AZ] M. Artin and J. Zhang, Noncommutative projective schemes, Adv. Math., 109 (1994), 228-287. MR 96a:14004

[CE] H. Cartan and S. Eilenberg, Homological Algebra, Princeton University Press, Princeton, 1956. MR 17:1040e

[D] I. Dolgachev, Weighted projective varieties, in Group Actions on Vector Fields (Vancouver, BC 1981), Lecture Notes in Mathematics No. 956, Springer-Verlag, 1982, pp. 34-71. MR 85g: 14060

[Ha] R. Hartshorne, Algebraic Geometry, Springer-Verlag, New York, 1977. MR 57:3116

[L] T. Levasseur, Some properties of non-commutative regular rings, Glasgow J. Math., 34 (1992), 277-300. MR 93k:16045

[LS] T. Levasseur and J. T. Stafford, The quantum coordinate ring of the special linear group, J. Pure Appl. Algebra, 86 (1993), 181-186. MR 94d:16038 
[Mu D. Mumford, Varieties defined by quadratic relations, in Questions on Algebraic Varieties, Ed. E. Marchionne, C.I.M.E, III Ciclo, Varenna 1969, Edizione Cremonese, Rome, 1970, pp. 29-100. MR 44:209

[Ro] J. J. Rotman, An Introduction to Homological Algebra, Academic Press, New York, 1979. MR 80k: 18001

[ST] J. Silverman and J. Tate, Rational Points on Elliptic Curves, Springer-Verlag, New York, 1992. MR 93g: 11003

[SS] S. P. Smith and J. T. Stafford, Regularity of the four dimensional Sklyanin algebra, Compositio Mathematica, 83 (1992), 259-289. MR 93h:16037

[Sn] R. L. Snider, Noncommutative regular local rings of dimension 3, Proc. Amer. Math. Soc., 104 (1988), 49-50. MR 89i: 16023

[SZ] J. T. Stafford and J. Zhang, Homological properties of (graded) Noetherian PI rings, J. Algebra, 168 (1994), 988-1026. MR 95h:16030

[St1] D. R. Stephenson, Artin-Schelter Regular Algebras of Global Dimension Three, Ph. D. Thesis, University of Michigan, UMI, 1994.

[St2] D. R. Stephenson, Artin-Schelter regular algebras of global dimension three, J. Algebra, 183 (1966), 55-73. CMP 1996:14

[W] Wall, C. T. C., Generators and relation for the Steenrod algebra, Annals of Math, 72 (1960), 429-444. MR 22:7117

Department of Mathematics, University of California, San Diego, San Diego, CaliFORNIA 92093

E-mail address: dstephen@math.ucsd.edu 